2018-10-01

\title{
Determination of Kinetic Parameters and Metabolic Modes Using the Chemostat
}

\author{
Boden, Rich
}

http://hdl.handle.net/10026.1/12441

10.1007/978-3-319-44535-9_24-1

Springer Nature Switzerland

All content in PEARL is protected by copyright law. Author manuscripts are made available in accordance with publisher policies. Please cite only the published version using the details provided on the item record or document. In the absence of an open licence (e.g. Creative Commons), permissions for further reuse of content should be sought from the publisher or author. 


\title{
Determination of Kinetic Parameters and Metabolic Modes Using the Chemostat
}

\author{
Rich Boden and Lee P. Hutt
}

\section{Contents}

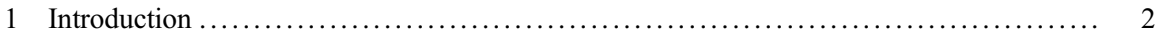

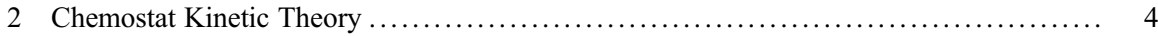

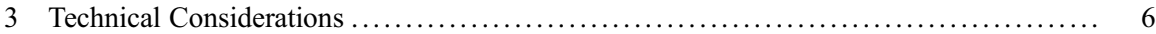

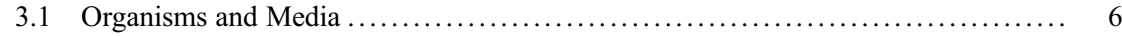

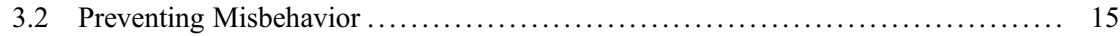

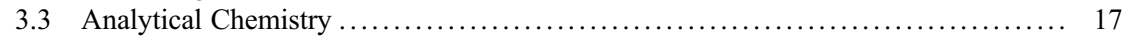

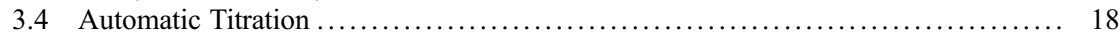

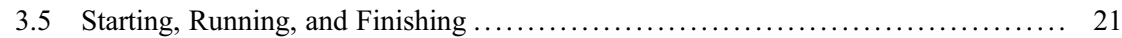

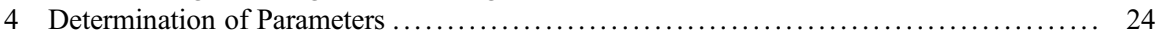

4.1 Maximum Specific Molar Growth Yield $\left(Y_{\mathrm{MAX}}\right)$ and Maintenance Coefficient $\left(m_{\mathrm{S}}\right) \quad 25$

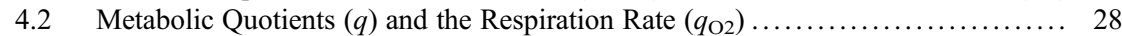

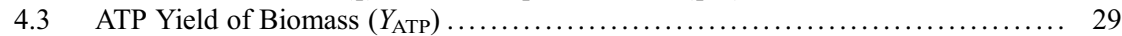

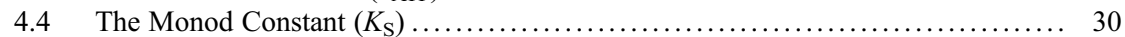

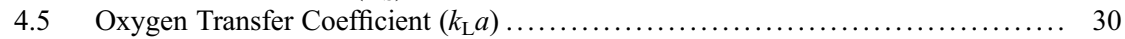

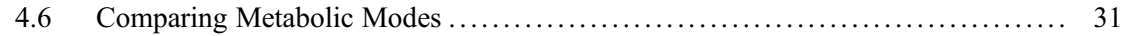

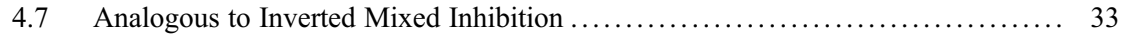

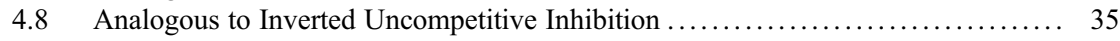

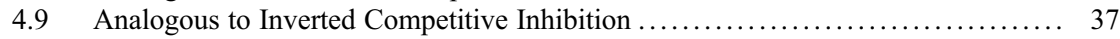

\footnotetext{
R. Boden $(\bowtie)$

School of Biological and Marine Sciences, Faculty of Science and Engineering, University of Plymouth, Plymouth, UK
}

Sustainable Earth Institute, Faculty of Science and Engineering, University of Plymouth, Plymouth, UK

e-mail: rich.boden@plymouth.ac.uk

\section{P. Hutt}

School of Biological and Marine Sciences, Faculty of Science and Engineering, University of Plymouth, Plymouth, UK

e-mail: lee.hutt@plymouth.ac.uk 


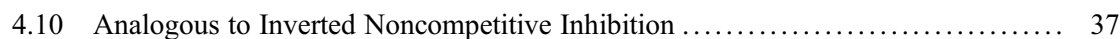

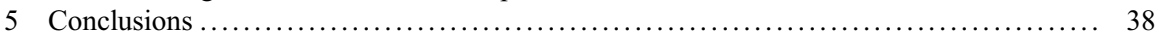

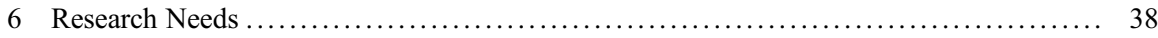

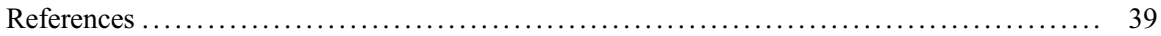

\begin{abstract}
Continuous cultures in the form of chemostats limited by carbon source, energy source, nitrogen, phosphate, or oxygen are commonly used where reproducible growth or a continuous supply of biomass is needed. Originally, they were developed as means to determine a range of kinetic parameters such as specific molar growth yield $(Y)$ and derived parameters such as maximum specific molar growth yield $\left(Y_{\mathrm{MAX}}\right)$, maximum specific growth rate $\left(\mu_{\mathrm{MAX}}\right)$, maintenance coefficient $\left(m_{\mathrm{S}}\right)$, specific maintenance rate $(a)$, the Monod constant $\left(K_{\mathrm{S}}\right)$, etc. These parameters afford very useful means of comparing organisms or determining metabolic modes within an organism but are sadly no longer widely used, largely for reasons of skill sets disappearing. In this chapter we cover the basics of running a chemostat to give high-quality growth data and manipulation thereof to obtain growth kinetic parameters.
\end{abstract}

\title{
$1 \quad$ Introduction
}

The most commonly used form of growth of Bacteria and Archaea in liquid culture is the batch culture, a closed system in which a finite amount of electron donor (which is also the carbon source, for growth of heterotrophs) is supplied in some manner of liquid medium. The organism grows until some nutrient runs out - either the carbon source or a trace metal, commonly zinc - or a toxic or inhibitory byproduct of growth builds up in the medium (e.g., hydroxonium ions or "protons" as we conventionally simplify to in biology). Batch cultures can also be undertaken in bioreactors ("fermenters" in common lab parlance, regardless of their metabolic mode) that afford larger volumes along with $\mathrm{pH}$ control, oxygenation control, stirring, etc.

Batch culture has a number of limitations, viz.:

- Cell "happiness" - for wont of a better word! When cells are grown in a closed system such as batch culture, they are swimming in their own waste - be that protons, fatty acids, side products, secondary metabolites, etc. - and this can cause metabolic stress and raise the maintenance needs of the cells, leading to low enzyme activities and low protein yields.

- Inability to change one variable - for example, if one wanted to examine expression of a gene that one thinks may be involved in temperature tolerance, one could grow shake-flask batch cultures or batch bioreactors at the optimum growth temperature and $5{ }^{\circ} \mathrm{C}$ higher. In changing the temperature, one also changes the specific growth rate $(\mu)$ as the hotter flask will be growing slower. 
Does the gene expression change owing to temperature or growth rate? One cannot elucidate this in batch culture.

- Unrealistic carbon source/electron donor levels - in the batch culture, the organism is supplied with, e.g., $20 \mathrm{mM}$ acetate at the beginning of culture, and this goes down over time. Even in high-acetate environments such as peat bogs, concentrations do not usually exceed $1 \mathrm{mM}$ (Avery et al. 1999); thus one has to consider how environmentally or "real-world" relevant the data collected are, since the cells are swimming in their electron donor or carbon source.

- Limited range of parameters determinable - useful parameters concerning maintenance or normalized forms of yields that are directly comparable with organisms from other genera or even other domains cannot be determined from batch cultures.

Various types of continuous culture exist, and most are based around vessels with some manner of input and output (the latter can be a weir in the wall or a drop tube set at a given depth with a pump attached) to maintain a constant volume - for every $\mathrm{mL}$ that enters the system, an $\mathrm{mL}$ leaves the system. Of the variations that exist, some serve to keep the cell number constant in the vessel at all times (turbidostat, cytostat) or to keep the level of cellular substrate consumption constant (auxostat) or the concentration of carbon source or electron donor constant (nutristat). In a system that keeps a given limiting substrate (be that the carbon source, electron donor, nitrogen source, phosphorus source, or even a metal) absent from the vessel at all times since it is wholly consumed, the amount of biomass is also constant when the system is in steady state - this is the chemostat.

Chemostat setups vary enormously and can be as basic as a sidearm test tube with medium dripped in from a Pasteur pipette pushed through a foam bung - while such systems of c. $50 \mathrm{~mL}$ volume are excellent teaching tools and can be handy for keeping "fussy" organisms alive, they are not suited to the much more rigorous conditions needed in the research laboratory. Chemostat setups of such rigor have been reported with volumes on the $\mu \mathrm{L}$ scale using microfluidics (Groisman et al. 2005; Long et al. 2013), with devices printable using stereolithography methods. Semimicro setups with volumes of as little as $10 \mathrm{~mL}$ can be produced by a glassblower with relative ease and can, with the right pumps, etc., allow a laboratory to run dozens of experiments at the same time (e.g., the Pirtian Mini-Loop system is very useful for aerobic phototrophs in particular, Pirt et al. 1979). The more typical systems are purpose-built, well-controlled devices that can be procured from specialist suppliers and which have a range of vessels that can hold cultures of $0.1-10 \mathrm{~L}$. Large chemostat volumes can be useful when the goal is to produce a steady-state culture and sacrifice it for protein purification, but for kinetic work, a small culture volume is much more convenient as it reduces the amount of time and medium consumed during equilibration. In our laboratory, we use $c .2500 \mathrm{~mL}$ vessels with $c$. $500 \mathrm{~mL}$ medium for kinetic studies, and we find this very convenient, with the large headspace being particularly useful when growing organisms on more challenging (to handle) substrates that require a long batch culture phase without air sparging, such as carbon disulfide - e.g., the $c .2000 \mathrm{~mL}$ volume of air contains about $17 \mathrm{mmol}$ 
oxygen, which is enough to oxidize about $8 \mathrm{mmol}$ carbon disulfide without the need to sparge the culture - headspace volume is an important consideration in the setting up of a reactor, which we discuss in due course.

In this chapter we will cover the theory of Monodian and Pirtian kinetics that underpins cultivation in the chemostat and the methodologies for determination of these parameters - we have split this into two separate sections with a technical section on how to actually do it all in between so that the reader can make proper consideration of ensuring their cultivation method will provide them with the data that they need that those data will not be subject to artifacts.

\section{Chemostat Kinetic Theory}

The basic parameters of the chemostat that require consideration are that a culture of $V(\mathrm{~mL})$ volume is contained in a vessel with an inflow and outflow. Fresh medium containing the limiting substrate of concentration $S(\mathrm{mM})$ is pumped into the vessel at a flow rate of $F(\mathrm{~mL} / \mathrm{h})$. The hydrodynamic dilution rate of the culture $D\left(\mathrm{~h}^{-1}\right)$ can be determined since:

$$
\boldsymbol{D}=\boldsymbol{F} / \boldsymbol{V}
$$

If the growth rate of the organism in the culture $\mu\left(\mathrm{h}^{-1}\right)$ - the rate of cell division is slower than $D$, the number of cells leaving in the outflowing medium will exceed the number of new cells from cell division; thus the culture turbidity will fade and the culture will "wash out." If $\mu$ exceeds $D$, more cells will be produced in the reactor than will leave in the outflowing medium; thus the culture turbidity will increase. The latter scenario is very common in the early stages of a continuous culture, but eventually $\mu$ will equal $D$, and a steady state will form in which the cell number remains constant - every cell division owing to substrate entering the vessel is balanced by a cell leaving in the outflowing medium. In this state, [substrate] is zero, not "below the detection limit," not " $<0.01 \mathrm{mM}$," but absolutely $0.000 \mathrm{mM}-$ since that is the very definition of the chemostat: a steady-state culture at which the limiting substrate is absent in the vessel. In such a condition, every molecule of the substrate that falls into the vessel is immediately consumed and immediately permits just enough growth to occur to balance to loss of cells. This gives a more environmentally relevant scenario since in many environments, a substrate may not be detectable, but the flux can be very high, as is the case in the chemostat at steady state (cf. oxygen in activated sludge). Many useful parameters can be determined from a chemostat culture, and they will be discussed in Sect. 4.

For chemostat theory to apply absolute, there are conditions that must be met:

(1) All growth must be planktonic and without foam. Wall growth or biofilms forming on probes, etc., will prevent a true, stable steady-state culture from forming. Foam rafts form owing to lysed cells, biosurfactant buildup, etc., and are more common in cultures growing on lipids, fatty acids, or hydrocarbons than on low-concentration sugars or inorganics - they cause issues with 
maintaining a constant volume and can also provide a surface for biofilms to colonize when they dry on the vessel wall. Silicone antifoam agents can control or prevent foam, and both this and mechanisms to prevent wall growth and biofilms are covered in Sect. 3.

(2) The culture must be very well mixed. Effectively "infinite mixing" of the culture is an absolute necessity, since the drops of substrate-bearing medium falling into the vessel must be disbursed instantaneously such that their consumption is instantaneous.

(3) Aside from the limiting substrate, everything else must be in excess. That is to say that metals, nitrogen, phosphorus, sulfur, etc., must all be in excess but so too must be the terminal electron acceptor - if this is molecular oxygen, then gassing must be very vigorous. In cultures of chemolithoautotrophs, it is important to insure that the limiting electron donor at, e.g., $20 \mathrm{mM}$ is truly limiting by ensuring a vast supply of carbon dioxide/(bi)carbonate.

(4) The medium must be entirely defined and should contain a single carbon source or a single electron donor. A basal salt medium, usually with a phosphate buffering system, is used and is supplemented with the carbon source or electron donor. While vitamins may be provided to ensure they are not limiting growth, there should not be any carbon sources present. It is not suitable to have any complex components in the medium, e.g., brain-heart infusion is commonly used in batch cultures at concentrations of $0.002 \%(w / v)$ when growing autotrophs as a "vitamin" - in the chemostat, using proper vitamin solutions is very important as they are fully defined and cannot be taken up as cosubstrates to produce biomass. It is not necessary to use a water-soluble limiting substrate - alkanes, elementary sulfur, etc., can be used.

(5) All parameters must be tightly controlled with feedback systems. For example, a thermistor probe in the culture feeds back to a microprocessor control unit that has been programmed by the user to hold the culture at $43{ }^{\circ} \mathrm{C}$. If the temperature momentarily drops owing to a draft or cool medium being fed in, the control unit turns on a heated probe or jacket and briefly warms the culture. If the culture becomes too warm owing to biogenic heat, the control unit opens a solenoid valve, running cold water through a cold finger to cool the culture. Similar systems are required for proton concentration (a $\mathrm{pH}$ probe and reservoirs of acid and base) and dissolved oxygen $\left(\mathrm{dO}_{2}\right)$ concentration as a minimum (for anaerobes, a redox potential $\left(E_{h}\right)$ probe may be more useful). To maintain culture volume, a Liebig or Allihn condenser is attached to the outflowing airline so that water vapor is returned to the vessel - at higher temperatures $\left(>50{ }^{\circ} \mathrm{C}\right)$, a Dimroth or Friedrichs condenser may be necessary as the efficiency will need to be higher. Often tap water is used to feed these condensers, but for hightemperature work, a chilling unit that can supply water at $2{ }^{\circ} \mathrm{C}$ is much more effective.

(6) The limiting substrate, other media components, and the production of biomass must be accurately quantifiable. It is quite impossible to run a chemostat if one cannot quantify the depletion of the limiting substrate - and for that matter, the phosphorus and nitrogen sources (to ensure they are not 
limiting) - and the production of biomass. For some electron donors that are difficult to measure without specialist equipment, such as dimethyl sulfone $\left(\mathrm{DMSO}_{2}\right)$, but for which the end products (sulfate) are stoichiometric and measurable, one can effectively measure substrate by proxy, as one can measure the sulfate being produced (Boden et al. 2011a; Borodina et al. 2000). It is important that all analytical work be done with great care and precision, appropriate glassware, etc. There is an emphasis on, e.g., kits provided by manufacturers for use by biologists to be high-throughput and fast, often working in very small volumes in plate readers where tiny pipetting errors change the path length; it is prudent to work like an analytical chemist with proper volumetric glassware and proper calibrants and - for colorimetric assays - using the largest path length cuvettes possible so as to extend accuracy.

\section{Technical Considerations}

In this section we discuss the setup and running of the chemostat as well as its rescue if things go awry.

\subsection{Organisms and Media}

Organisms wont to forming biofilms, rosettes, etc., can be difficult to manage in the chemostat in terms of keeping all growth planktonic, but we give herein methods for preparing glassware to avoid biofilm formation, and rapid stirring is often sufficient to reduce clumping of cells.

\subsubsection{Biomass Determination}

It is critical that the amount of biomass is determined accurately and that optical density is not used as raw data, but is converted to a meaningful unit. In our hands, both colorimeters and spectrophotometers are equally useful for biomass determination as long as they are well calibrated. Many users use an arbitrary wavelength and determine optical density $(O D)$ at, e.g., $600 \mathrm{~nm}\left(O D_{600}\right)$ without giving it much thought, but it is absolutely critical that proper calibrations are made for every organism used, (1) to determine the proper wavelength to use and (2) to determine the $O D$ conversion to biomass factor. This is achieved by growing flasks of the organism on various substrates (since accumulation of volutin, etc., can alter the results) to late-exponential phase. Cells are harvested, washed, and resuspended in the same basal medium they were grown in or in a suitable buffer and are then diluted to a range of at least five arbitrary turbidities $-3 \times 1 \mathrm{~L}$ of each turbidity is required, and they are diluted in volumetric flasks - the optical density of each being checked in replicate cuvettes at a range of wavelengths from $440 \mathrm{~nm}$ to $740 \mathrm{~nm}$ (below $440 \mathrm{~nm}$ the Soret peak of cytochrome $c$ tends to cause interference - we usually use 440, 540, 640, and $740 \mathrm{~nm}$, as a minimum) then returned to the flask. A set of $0.2 \mu \mathrm{m}$ filters ( $45 \mathrm{~mm}$ diameter) are placed in all-glass filter holders on top of 
$0.45 \mu \mathrm{m}$ filters as supports and are dried overnight at $95{ }^{\circ} \mathrm{C}-$ this is necessary to remove any moisture and must be done within the holders; otherwise, they curl and then crack when used. The filters are removed and weighed on an analytical balance and replaced in the holders. Each is used to filter one of the replicate turbidities, and the cells on the filter are then washed with $500 \mathrm{~mL}$ of the same basal medium, then with $100 \mathrm{~mL}$ ice-cold water. Filters are dried in crystallizing dishes at $70-95{ }^{\circ} \mathrm{C}$ to constant mass (up to a week - a vacuum oven can speed this up) and are weighed. From the mean amount of biomass at each optical density, one can plot the $O D$ amount of biomass (ordinate, no units) against the concentration of biomass (abscissa, mg dry biomass/L) with different lines for each $O D$ wavelength used. From these data, the linearity of the data can be examined - we usually look for one linear to $O D=0.9$ as we routinely dilute all samples $>0.9$ for analysis anyway since spectrophotometer inaccuracy begins to interfere. The conversion ratio (e.g., for Thermithiobacillus tepidarius strains, $O D_{440}=0.1$ is $23 \mathrm{mg}$ dry biomass per L) can then be determined. In Fig. 1, we show representative data for Pseudomonas, Achromobacter, and Thermithiobacillus - it can be seen that in all cases, $440 \mathrm{~nm}$ permits the largest change in $O D$ per unit dry biomass, thus affords the most useful data. Koch (1981) also found that low wavelengths were also the most useful for small cell sizes. The linearity of the data does vary, particularly for Pseudomonas and Achromobacter at low densities, but the standard error of mean is usually consistently small within each organism's data. The exception to this is the Thermithiobacillus sp., which has greater standard error of mean, potentially owing to carboxysomes ("polyhedral bodies") present in the cells being at variable number and the very high cytochrome $c$ content of sulfur-oxidizing Bacteria, for which the tail end of the Soret peak will possibly give some minor interference at $440 \mathrm{~nm}$ and the beginning of the $\alpha$ peak will possibly interfere at $540 \mathrm{~nm}$, but these absorbances are usually very low versus optical densities, and the latter has the greater impact on the value obtained.

Some workers use the amount of protein or amount of DNA in lieu of the amount of dry biomass, but these are very prone to change during growth and should be avoided. Cell carbon is a useful unit if one has access to a total organic carbon (TOC) analyzer or can have CHNS analyses performed on a sample, but we have found for various members of the Proteobacteria that they contain $48 \% \mathrm{C}$ by mole in every case. Cell number is quite a useless unit in chemostat kinetics since 20 small cells could have the same amount of biomass as five large cells or the culture could contain a range of cell sizes in a pleomorphic organism; thus we strongly recommend dry biomass or cell carbon aredetermined.

\subsubsection{Protein Determination}

While we strongly recommend cell protein not be used as the unit of growth, it can be useful to determine as a secondary factor, and the bicinchoninic acid assay (Smith et al. 1985) is generally the most useful; however, calibration should be carefully considered since the de rigueur bovine serum albumen does not always give a particularly good calibration. Microtiter plate assays should be avoided as the path length is not consistent $-1 \mathrm{~mm}$ path length optical glass cuvettes may be a slight 

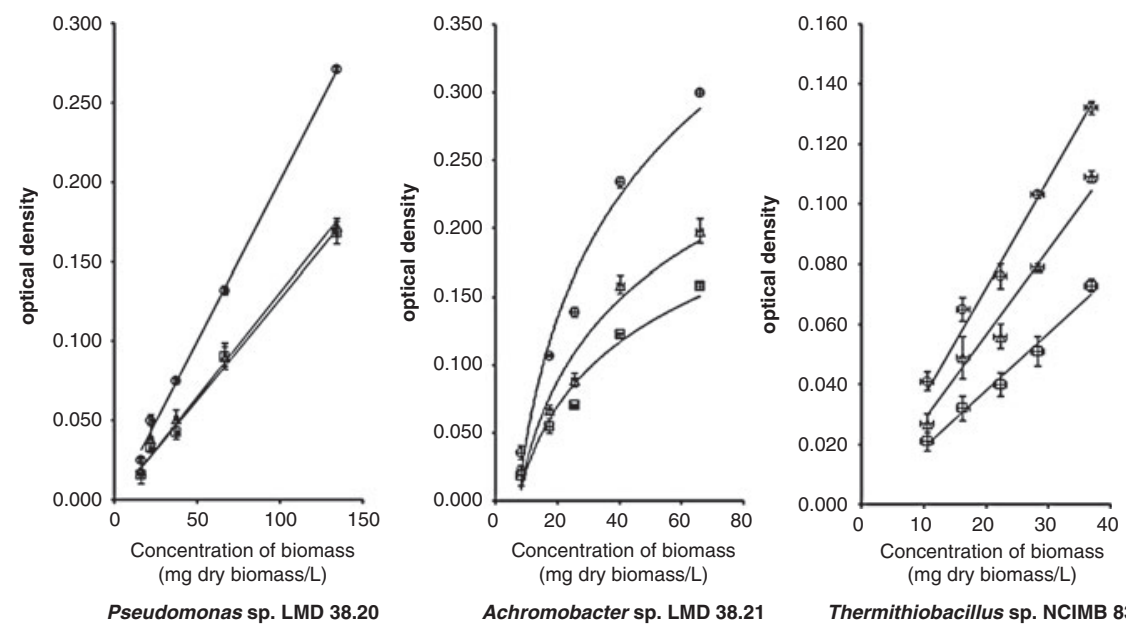

Fig. 1 Relationships between concentration of dry biomass and optical density at a range of wavelengths for members of the genera Pseudomonas (Gammaproteobacteria), Achromobacter (Betaproteobacteria), and Thermithiobacillus (Acidithiobacillia). Optical density determination wavelengths are indicated by circles $(440 \mathrm{~nm})$, triangles $(540 \mathrm{~nm})$, and squares $(600 \mathrm{~nm})$. Error bars are standard error of mean $(n=3)$. Briefly, cells obtained from glucose, succinate, or thiosulfate-limited chemostats (respectively) were washed in phosphate-buffered saline to give a range of turbidities. Optical density was determined at three wavelengths for each suspension before drying to constant mass at $70{ }^{\circ} \mathrm{C}$ in pre-weighed glass scintillation vials versus a cell-free control. None of the organisms produced endospores, exospores, or cysts. They do not contain stacked internal membranes nor do they produce intracellular volutin (polyphosphate), lipid ( $\beta$-polyhydroxybutyrate), or polysaccharide granules. Thermithiobacillus sp. NCIMB 8349 contains carboxysomes (polyhedral bodies) at variable numbers per cell in common with other members of the genus (cf. Boden et al. 2016) but does not produce elementary sulfur granules in continuous culture (Hutt 2016). Cell were short slender rods - Pseudomonas sp. LMD $38.20(0.5 \times 2.6 \mu \mathrm{m})$, Achromobacter sp. LMD $38.21(0.8 \times 1.2 \mu \mathrm{m})$, and Thermithiobacillus sp. NCIMB 8349 $(0.5 \times 1.5 \mu \mathrm{m})$

inconvenience, but they are more precise. For calibration, proteins are best extracted at the French press and debris not removed, but the lysate is then treated with trichloroacetic acid (TCA) for which many protocols exist and the protein precipitate washed in acetone and redissolved before determination - this removes almost all interfering factors.

\subsubsection{Cell Counting}

Many workers count cells even though this is not a useful parameter for kinetic work really. This can be done by flow cytometry or using a Neubauer hemocytometer whatever is used, vital or live-dead staining must be used to separate biomass from necromass - many commercial kits based on fluorescent dyes such as SYTO $^{\circledR} 9$ and propidium iodide are available. Alternatively dyes such as $N$-(3-triethylammoniumpropyl)-4-( $p$-diethylaminophenyl-hexatrienyl) pyridinium dibromide (FM 4-64) can be used in "DIY" approaches (Pogliano et al. 1999). A complex of 
Ponceau S (Acid Red 112) and depleted uranyl sulfate or uranyl acetate can be used for vital staining of some unicellular Eukarya and Bacteria - we have used it with Methylobacterium spp. - and it can be visualized with a normal microscope (van Steveninck and Booij 1964). Sunset Yellow FCF (Orange Yellow S) has recently been reported as a "vital stain" for the Bacteria although the original publication does not show any live-dead staining of cells - this may simply be a stain that does not kill the cell, rather than being a true vital stain (Gao et al. 2017).

\subsubsection{Basal Media}

In this section we cover preparation of media and some example media that we use in our laboratory. For the very best data to be obtained, analytical grade chemicals should be used for all media components and for the limiting substrate and for the acid and base used for $\mathrm{pH}$ control - while this is costly, the data quality is worth it, and after the initial outlay, the chemicals will keep for many batches of medium.

Media for the chemostat should be made in glass-distilled water (or equivalent we have found amino acid contamination in "Milli-Q" water, however) and use Class A volumetric glassware to minimize variation. All carboys and glassware should be cleaned by soaking overnight in $2 \%(v / v)$ nitric acid and then washing in glass-distilled water until the washings are neutral to $\mathrm{pH}$ paper. To make, e.g., a 20 L Nalgene carboy of medium that requires phosphates to be autoclaved separately in $25 \%$ of the final volume, the phosphate stock is made up of a $5 \mathrm{~L}$ Class A volumetric flask and is autoclaved in a $10 \mathrm{~L}$ carboy that has a one-port lid with a large $0.2 \mu \mathrm{m}$ pore size filter attached, which is wrapped in foil for autoclaving. The ingredients for the remainder of the medium are added to the $20 \mathrm{~L}$ carboy with a very large glass-coated "flea" and $15 \mathrm{~L}$ glass-distilled water is added in the form of $3 \times 5 \mathrm{~L}$ volumetric flasks as before. The carboy is stirred until everything has dissolved and placed on a level bench (checked with a spirit level), and once settled, a very fine permanent marker is used to mark the bottom of the meniscus at intervals around the vessel. The lid of the vessel should have three ports - one is connected to a drop tube leading to the bottom of the vessel - this is silicone tubing that is weighted with a short length of glass tubing at the lower end to keep it in the medium and prevent it from floating. The outside of this port is connected to silicone tubing and terminated with tubing connector, which is wrapped in foil. This tube is clamped off with Spencer Wells artery forceps or with gate clamps - we use the former as they are easier to operate with one hand. The next port has no interior connection, and the exterior is connected to a large diameter $0.2 \mu \mathrm{m}$ pore size filter, which is wrapped in foil - this connection must not be clamped when autoclaved! The final port is connected to the bottom sidearm of a large aspirator bottle or $1 \mathrm{~L}$ or $2 \mathrm{~L}$ volume, using a long length of tubing that is terminated half way with a clamp and foil wrapped as before. The top of the bottle is plugged with a foam bung and is wrapped in foil. When autoclaving media, it is critical that the autoclave is used properly viz., that a second $20 \mathrm{~L}$ carboy is filled to the same volume with water and is autoclaved with a wad of cotton wool instead of the stopper and with the "load" thermistor of the autoclave sitting in the bottom of it - this ensures that the medium is properly heated for the right amount of time (usually $10 \mathrm{psi}$ for $15 \mathrm{mins}$, but it may 
take $12 \mathrm{~h}$ for a large volume to come to temperature): do not rely on autoclave tape as an indicator! If the medium is $<\mathrm{pH} 6.5$, it is important that no proteinaceous broths or wastes are autoclaved at the same time as their (poly)amine fumes may pull the $\mathrm{pH}$ of the medium up. Autoclaving a large funnel wrapped in foil is very useful for the next stage. The autoclaved medium and phosphate solution are allowed to cool fully usually overnight - we place them in the fume hood as the draft speeds up this process somewhat.

The $20 \mathrm{~L}$ carboy is positioned on a bench or on the floor, with the aspirator bottle on the bench close to a Bunsen burner. The level of the liquid in the carboy is checked against the line and carefully topped up with sterile distilled water if need be. Foil coverings on filters are removed, and aseptically, using the sterile funnel, the phosphate solution is poured into the aspirator bottle until it is full. The foam bung is put back in place and the clamp is then removed, and the bottle is raised to dispense phosphate into the medium carboy. This is repeated until all of the phosphate has been added. If any substrate or vitamin stock is to be added to the carboy after autoclaving, we add it to the aspirator within the first aliquot of the phosphate solution so that the later aliquots ensure it is fully washed in. The clamp between the aspirator and the carboy is tightly closed and the tubing severed on the aspirator side - razor blades are the best thing for cutting tubing as they give a straight cut. The carboy is then placed adjacent to the reactor on top of a magnetic stirrer, and it is stirred continually during use to prevent any sediments forming. The connection to the vessel and the determination of flow rate are covered in Sect. 3. For some extreme thermophiles, a stirring hotplate is useful to preheat the medium.

Basal media for use in the chemostat do not require vast buffering capacities, since the system is under automatic proton concentration control - being able to reduce this allows phosphate limitation to be undertaken with ease. For some work, using organic Good buffers (Good et al. 1966) is useful but with caution. Tris is always to be avoided - most autoclave-safe $\mathrm{pH}$ electrodes cannot determine $\mathrm{pH}$ in Tris-buffered solutions and can be damaged by it, plus there is a lot of variance of $\mathrm{pH}$ with temperature in this system. Tightly controlled batch culture work is needed to determine if they have any inhibitory effect or can be consumed as a carbon or nitrogen source. It is worth noting that piperazine- $N$ - $N^{\prime}$-bis(2-ethanesulfonic acid) (PIPES), 4-(2-hydroxyethyl)-1-piperazineethanesulfonic acid (HEPES), 3-[4-(2hydroxyethyl)piperazin-1-yl]propane-1-sulfonic acid (HEPPS), and piperazine- $N$ $N^{\prime}$-bis(2-hydroxypropanesulfonic acid) (POPSO) can form radicals and thus disrupt redox chemistry or oxidize some substrates and should be avoided (Grady et al. 1988). Bicine and tricine should also be avoided as they inhibit flavin and flavonedependent enzymes in the presence of light, during which they are oxidized themselves, thus lowering buffering capacity (Soni and Kapoor 1981). Consideration must also be made of any unwanted effects of buffers on downstream methods for quantification of the limiting substrate. A wider discussion of the full gamut of buffering systems including bicarbonate, Good buffers, succinate, etc., is given in Good and Izawa (1972).

We tend to use phosphate buffering, and our usual medium for continuous culture for non-halophiles is E-basal salts (EBS, modified from Kelly and Syrett (1964)) 
Table 1 Composition of E basal salts (EBS) for use in the chemostat as a generalist basal medium for aerobic organisms. The phosphates are autoclaved separately in $25 \%$ of the final volume and are combined once totally cold - the vitamin stock is added to the combined medium once cold. Trace metal solution T and vitamin solution VJK are given in Tables 2 and 3, respectively

\begin{tabular}{l|l}
\hline Component & Concentration $(\mathrm{g} / \mathrm{L})$ \\
\hline Potassium dihydrogen phosphate & 4.00 \\
\hline Dipotassium hydrogen phosphate & 4.00 \\
\hline Ammonium chloride & 0.40 \\
\hline Magnesium sulfate heptahydrate & 0.80 \\
\hline Trace metals solution "T" & $10.00 \mathrm{~mL}$ \\
\hline Vitamin solution "VJK" & $0.10 \mathrm{~mL}$ \\
\hline
\end{tabular}

which is given in Table 1. EBS can be used as is with up to $60 \mathrm{mM}$ substrate carbon (e.g., $10 \mathrm{mM}$ glucose) under carbon limitation. For phosphate limitation, the two phosphate salts are reduced to $0.4 \mathrm{~g}$ each. For nitrogen limitation, we increase the substrate carbon to $90 \mathrm{mM}$ total. If used with chemolithoautotrophs, $20 \mathrm{mM}$ thiosulfate or $10 \mathrm{mM}$ tetrathionate, etc., can be used in place of the carbon source for electron donor limitation. The $\mathrm{pH}$ of EBS is 7.0 but can be varied from $\mathrm{pH} 5.0$ to $\mathrm{pH} 8.5$ by varying the phosphate ratios on the basis of the standard buffer tables provided in most biochemical and chemical texts. The trace metals solution " $\mathrm{T}$ " is modified from Tuovinen and Kelly (1973) and is given in Table 2. It is useful to model the solution chemistry of defined media during autoclaving, cooling, and mixing to ensure that the final solution concentrations of all components are sufficient for growth and to understand the composition of any precipitates that may form - PHREEQC (Parkhurst and Appelo 1999) is a very useful platform for this and can give important insight, particularly when considering critical trace metal ions such as $\mathrm{Cu}$ (II) versus $\mathrm{Cu}(\mathrm{I})$ versus $\mathrm{Cu}$ mineral speciation in media for the growth of methanotrophs or when studying cytochrome $c$ oxidases.

We discuss issues with foam in Sect. 3.2.1, but in the absence of a foam controller, it is prudent to add a non-emulsion non-silicone antifoam such as Antifoam 289 or Antifoam 204 (Sigma-Aldrich) to each carboy before autoclaving at a final concentration of about $0.002 \%(v / v)$ - these should be checked in batch culture to determine if they have any inhibitory effects on the organism.

For the acid and base solutions required for the autotitrator to maintain proton concentration, these are usually "matched" to the medium - e.g., for EBS we would usually use $0.500 \mathrm{M}$ sulfuric acid and $1.000 \mathrm{M}$ potassium hydroxide as EBS is high in sulfate and potassium already. For autotrophs growing under electron donor limitation on, e.g., thiosulfate, where sulfuric acid is produced by the culture, we use $1.000 \mathrm{M}$ potassium carbonate so that the dissolved inorganic carbon (DIC) in the culture is kept high and non-limiting. All acid and base solutions should be made in volumetric flasks and standardized by titration against a standard acid or base purchased - as we do - at a precise concentration rather than made up in the laboratory - this can be surprisingly economical as many suppliers hold annual sales of titration supplies, including standardized acid and base solutions. For 
Table 2 Composition of trace metal solution T, modified from Tuovinen and Kelly (1973). The sodium hydroxide is dissolved in about $400 \mathrm{~mL}$ glass-distilled water, and the disodium EDTAate is added. Once dissolved, each of the remaining ingredients is added, in order, after dissolving in about $40 \mathrm{~mL}$ glass-distilled water, with washings. The solution is then adjusted to $\mathrm{pH} 6.0$ using $1 \mathrm{M}$ sodium hydroxide solution (20-25 mL) added slowly with stirring and is then diluted to $1 \mathrm{~L}$ in a volumetric flask. It is stored at room temperature in non-actinic glass, and the color may vary from green to red without any effect on performance

\begin{tabular}{l|l}
\hline Component & Concentration $(\mathrm{g} / \mathrm{L})$ \\
\hline Sodium EDTAate dihydrate & 50.00 \\
\hline Sodium hydroxide & 9.00 \\
\hline Zinc sulfate heptahydrate & 5.00 \\
\hline Calcium chloride & 5.00 \\
\hline Manganous chloride tetrahydrate & 2.50 \\
\hline Cobaltous chloride hexahydrate & 0.50 \\
\hline Ammonium heptamolybdate tetrahydrate & 0.50 \\
\hline Ferrous sulfate heptahydrate & 5.00 \\
\hline Cupric sulfate pentahydrate & 0.20 \\
\hline
\end{tabular}

determination of the precise concentration of potassium hydroxide solutions, one would titrate against standardized 1.000 M hydrochloric acid using phenolphthalein; for potassium carbonate, the same acid is used with methyl orange. For sulfuric acid, use standardized $1.000 \mathrm{M}$ sodium hydroxide and phenolphthalein. The great advantage of standardizing is the absolute ability to determine how much acid/base was consumed by the chemostat, thus how much was produced per mole of substrate, ultimately. This is particularly useful in fermentations and in growing sulfur autotrophs.

\subsubsection{Vitamin Solutions}

There are many published vitamin stock solutions in common use, such as the one we give in Table 3. This solution is light and heat labile when concentrated and should only be added to media after autoclaving. For truly auxotrophic organisms (e. g., Thiomicrospira pelophila DSM $1534^{\mathrm{T}}$ for vitamin $\mathrm{B}_{12}$ ), individual vitamins should be added which is both less expensive and less wasteful. Our practice is that for all organisms, we use a vitamin solution at $0.1 \mathrm{~mL} / \mathrm{L}$ as this ensures nothing can run out and become limiting during growth.

\subsubsection{Carbon Sources}

If it exists, it is probably a carbon source for something. Carbon sources can be solids, liquids, or gases at room temperature and have varying levels of solubility.

For gaseous carbon sources, two options are available - one is for, e.g., monomethylamine (MMA), where a solid hydrochloride salt exists and can be dissolved in media instead. For gases that do not have soluble salts, e.g., methane, the only option re: a chemostat is to limit by oxygen, phosphate, or nitrogen as methane limitation is very hard to achieve, technically. For an, e.g., phosphate-limited system, the chemostat is sparged with air at about $2 \mathrm{~L} / \mathrm{min}$ for a $500-1000 \mathrm{~mL}$ culture, and the 
Table 3 Composition of vitamin solution VJK, given to us by Dr. Jan Kuever, MPA Bremen (Germany), and which we have used for some time. The vitamins are added in order either directly or from stock solutions to about $400 \mathrm{~mL}$ glass-distilled water. Once all have dissolved, the $\mathrm{pH}$ is very carefully adjusted to $\mathrm{pH} 4.0$ using $0.1 \mathrm{M} \mathrm{NaOH}$ and is then diluted to $1 \mathrm{~L}$ in a volumetric flask. The solution is sterilized by passage through a $0.2 \mu \mathrm{m}$ filter and is stored in non-actinic glass at $4{ }^{\circ} \mathrm{C}$. It is our practice to store it as many $50 \mathrm{~mL}$ aliquots, which are used by individual workers, and prevent contamination of a large batch of very costly solution

\begin{tabular}{l|l}
\hline Component & Concentration $(\mathrm{mg} / \mathrm{L})$ \\
\hline Cyanocobalamin $\left(\mathrm{B}_{12}\right)$ & 100 \\
\hline$p$-Aminobenzoic acid $\left(\mathrm{B}_{10}\right)$ & 80 \\
\hline Biotin $\left(\mathrm{B}_{7}\right)$ & 20 \\
\hline Nicotinic acid $\left(\mathrm{B}_{3}\right)$ & 200 \\
\hline Calcium pantothenate $\left(\mathrm{B}_{5}\right)$ & 100 \\
\hline Pyridoxine hydrochloride $\left(\mathrm{B}_{6}\right)$ & 300 \\
\hline Thiamine hydrochloride $\left(\mathrm{B}_{1}\right)$ & 200 \\
\hline Lipoic acid & 50 \\
\hline Riboflavin $\left(\mathrm{B}_{2}\right)$ & 50 \\
\hline Folic acid $\left(\mathrm{B}_{9}\right)$ & 20 \\
\hline
\end{tabular}

air feed is supplemented with methane at $20-30 \mathrm{~mL} / \mathrm{min}$. Note that for methane specifically, during the first $24-48 \mathrm{~h}$ of growth, carbon dioxide should also be added, at $20 \mathrm{~mL} / \mathrm{min}$, but this is not needed once the culture is established.

For water-soluble solids and liquids, if they are stable and will remain in solution during autoclaving (e.g., glucose or glycerol), they can be added to the medium carboy. If they are likely to volatilize (e.g., methanol) or degrade (e.g., cysteine), they should be filter-sterilized as a stock solution and added to the cooled medium with the phosphates. For some very pungent and/or toxic volatile organics (e.g., dimethylsulfide, carbon disulfide), special procedures must be adhered to, which we describe elsewhere. For less soluble solids and liquids, they can be added to very low concentrations or added until they saturate the medium completely.

For very unstable compounds, it is prudent not to add them to the medium but to meter them into the reactor from a separate stock as described in Sect. 3.1.7.

\section{Carbon Sources for Generalist Heterotrophs}

When selecting appropriate substrates for "generalist" organisms, we have found it most useful to use intermediates of Krebs' cycle, if the organism will grow on them, such as succinate. This enables relatively easy determination of the theoretical yields. Batch culture experiments are needed to determine how fast it will grow, approximately, and what kinds of yield can be obtained. If the carbon source cannot be accurately quantified, it cannot be used. It is obviously important to have a firm view of what the pathway of dissimilation to carbon dioxide is so that theoretical yields can be determined. 


\section{Carbon Sources for Specialist Groups}

For methanotrophs, methane should be of very high purity as contaminating alkanes will kill the culture. For methylotrophs, very high purity substrates are needed - if formaldehyde is used, it should be made from the thermal depolymerization of paraformaldehyde (Chongcharoen et al. 2005) as procurement of methanol-free formaldehyde is very expensive and only small-volume ampoules are sold.

Dimethylsulfide (DMS) and dimethylsulfoxide (DMSO) should be from ultrapure stocks. The latter must be kept over molecular sieve to ensure that it does not absorb water. For volatiles like DMS, Viton tubing should be used throughout, and medium should be in a glass carboy as the substrate will escape through Nalgene.

Thiol stock solutions are made by adding to well-deoxygenated water (boiled while bubbling with argon, under reflux for 15 min with a drying tube on the top of the reflux condenser, then cooling fully under an atmosphere of dry argon). Most are liquid, but for the gaseous methanethiol, this is done by bubbling through the water in a Drechsel bottle and then destroying the "off-gas" by passage through household bleach (as thiols are extremely pungent and even if the work is done in the fume hood, the local streets will smell of it! There is a serious danger when working with thiols of causing panic of a gas leak as in many countries, and ethanethiol is added to the natural gas supply to give it a detectable smell - the first author speaks from experience of having done this!). A saturated solution made in this way is about $0.3 \mathrm{M}$ methanethiol, and this can be quantified and then diluted in medium (in a glass carboy, never Nalgene) appropriately before use. It will dimerize into dimethyl disulfide (DMDS) over time, and this must be monitored and the medium changed as necessary - a key point in kinetic chemostats is the need to monitor medium continually.

\subsubsection{Electron Donors for Chemolithoautotrophs}

Electron donors such as thiosulfate, sulfide, sulfite, and tetrathionate of high purity can be obtained from most chemical suppliers. Sodium polythionates must be synthesized by the user (Boden et al. 2010 gives details and references) as must dithionate (we give details elsewhere in this handbook - Boden and Hutt chapter > "Chemolithoheterotrophy - Means to Higher Growth Yields from This Widespread Metabolic Trait?"). Polythionates, sulfide, and sulfite are filter sterilized and added to media just before use - sulfide is usually neutralized with sulfuric acid to the same $\mathrm{pH}$ as the medium. We flush the headspace of the medium carboys with argon and attach a balloon of argon to the air filter on the carboy so that the headspace stays full of argon to minimize auto-oxidation, and this slight overpressure prevents leaks.

Elementary sulfur can be added as a Weimarn sol or a Reimi sol since a solid substrate is not practical (von Weimarn 1926; Raffo 1908; Steudel 2003). If the medium needs to be pumped into the reservoir at $F \mathrm{~mL} / \mathrm{h}$ to achieve the required $D$, when working with sulfur sols (which should be made aseptically in sterile carboys as they cannot be autoclaved), one should reduce the medium flow to $0.9 F$ and meter the sol into the vessel at $0.1 F$ using tubing with a very narrow caliber to minimize settling. This gives the same dilution rate but avoids issues of instability by keeping 
the sols away from the medium. It is wise to wash all glassware and plasticware overnight in concentrated hydrochloric acid if using the Weimarn sols as alkaline debris on the glass will make them less stable (Steudel 2003). The same "fractional flow rate" method can be used to add labile substrates such as sulfide or polythionates from separate stock solutions.

Carbon disulfide should be purified according to Skidmore (1979), and in the final step, the distillation should be done under argon and the collected product stored under argon at $-80{ }^{\circ} \mathrm{C}$ and then added to the medium just before use (in glass carboys). There will still be some oxidation to carbonyl sulfide, but purification in this way removes most of it, which has metabolic importance but also reduces the smell in use - purified carbon disulfide smells similar to diethyl ether, rather than of decomposing vegetables.

\subsection{Preventing Misbehavior}

A kinetic run of the chemostat can last some months if well-managed, but issues can and do arise - we strongly advise that foam, wall growth, and contamination contingencies are in place before a run starts - some substrates are very costly, and it is not worth wasting money, quite simply.

\subsubsection{Foam}

Foam can be both prevented and cured and we usually use both systems. For prevention, a low concentration $(0.002 \% v / v)$ of Antifoam 289 or Antifoam 204 (Sigma-Aldrich) added to the medium reservoir before autoclaving tends to prevent foam formation. Foam probes are comprised of an electrode in the medium and another in the lid of the vessel such that a foam raft would link them and complete an electrical circuit, which triggers a pump connected to a reservoir of antifoam. We use $0.5 \%(v / v)$ of the same antifoam agent in this reservoir - though some workers prefer the emulsion-based Antifoam A (Sigma-Aldrich) for the same purpose - our intention is that the foam probe and dosing system only operates in extremis and that the low level of antifoam in the medium is the main preventative measure. Pirt and Callow (1958) found that antifoam added as a bolus when foam rafts appeared actually led to more severe foaming and that antifoam added to the medium or meted into the vessel at regular intervals was superior. Whatever antifoam is used, it must be tested with the organism to ensure it does not inhibit growth.

\subsubsection{Wall Growth}

Growth on the walls of the vessel destroys the steady state and makes it very difficult to obtain any useful data during kinetic runs. For vessels with overflow weirs, biofilm growth in the weir can block the overflow and cause floods and damage to the equipment. This can be prevented by treating the glass to render it hydrophobic, but for some organisms like Hyphomicrobium spp., if they cannot colonize the glass, they will tend to form flocs in the culture, which can be just as difficult to obtain useful data from; however, very violent stirring ( $>400 \mathrm{rpm}$ ) combined with rapid 
aeration is usually enough to create so much turbulence that they are unable to aggregate into rosettes and larger structures.

Before use, after thorough washing with $2 \%(v / v)$ nitric acid, the vessel should be soaked overnight in glass-distilled water and then drained and wiped dry with lens tissue. Is it then coated with $0.15 \mathrm{M}$ dimethyldichlorosilane in octamethylcyclotetrasiloxane ("D4") or chloroform $(1.8 \mathrm{~mL}$ of the former is diluted to $100 \mathrm{~mL}$ with either solvent) on tissue (a pipe cleaner should be used to attend to overflow weirs) and left in the fume hood for $1 \mathrm{~h}$ (hydrogen chloride gas is evolved) before thoroughly washing with $1 \%(v / v)$ Neutracon ${ }^{\circledR}$ (Decon Laboratories Ltd, Hove, UK) and then deionized water and then autoclaving. This renders the glass hydrophobic even in 1-year runs (Boden et al. 2010, 2011a, b) and minimizes wall growth significantly. Domestic silicone glass treatments, such as Rain- ${ }^{\circledR}$ Rain Repellent (Illinois Tool Works (USA) or Kraco Enterprises (UK)), which is mostly polydimethylsiloxane, are potentially a significantly more economical alternative, and while we have used Rain- $X^{\circledR}$ Rain Repellent to coat glass plates for acrylamide gel casting (it works very well), we have not tried using it on culture vessels ourselves.

Biofilm growth in the tube from the vessel to the waste pot is also a cause of problems such as blockages and flooding, and this is easy to remove by massaging the tubing between the finger and thumb from top to bottom every day or every few days. This tubing must be very straight as any sag will harbor strong biofilm growth. If there is a continual flow of off-gas through this tubing (as there will be in most systems), this will usually prevent a lot of biofilm growth, but not all of it. If Viton or other opaque tubing is used, some short glass sections as "windows" will enable the worker to identify if biofilm is building up by massaging the region above the tubing and then watching for chunks of biofilm to wash down.

\subsubsection{Grow Back}

It is not uncommon for the medium feed line to get contaminated by cells splashing up from the vessel, and if minor, this can be ignored, but if it starts to grow back toward the medium reservoir, it becomes a major issue. Two main methods of prevention are in common use. A glasstundish, more commonly called an "antigrow-back trap," can be added to the medium feed just above the vessel. This comprises a glass bulb (spherical or cylindrical - usually about $2.5 \mathrm{~cm}$ diameter) with olives top and bottom, and the medium flow is simply broken by it dripping through an air space. This is our most commonly used option, but since they must be made by a scientific glassblower and the latter are becoming hard to find outside of major glassblowing companies (cf. Xia 2016) that charge a high premium on small jobs, they are not economical to produce individually - some basic glassblowing skills would allow the worker to make their own from a test tube and two pipettes without much effort - they don't need to look perfect; they just need to function! The second option also requires a glassblower unless a suitable pre-made product can be found. Leibig condensers of slightly wider caliber than the outside diameter of the medium feed are obtained and the socket and cone cut off so that they can be slide over the feed line before autoclaving. Afterward, hot silicone oil $\left(60{ }^{\circ} \mathrm{C}\right.$ for 
mesophiles) is recirculated through the glass jacket - this is enough to kill organisms trying to colonize the feed line, and the heat of the medium is dissipated during the drop into the vessel and landing in temperature-controlled medium. This same setup can be used to preheat medium for extreme thermophiles.

\subsubsection{First Aid Kit}

If the chemostat becomes contaminated, it is honestly best to abandon ship and just start over, but if the contamination is very low level, it can sometimes be rescued. We keep a "first aid kit" in the fridge comprising $10 \mathrm{~mL}$ syringes with 19 gauge syringe needles, loaded with sterile solutions of (a) $100 \times$ stocks of antibiotics that the organism under test can resist, (b) soluble antifoam at $10 \times$ the usual strength, and (c) $100 \times$ stock of the carbon source or electron donor based on double that at which is used in the basal medium. Between these, we can usually remedy low-level contamination by switching the reactor into batch mode and injecting antibiotics and monitoring the contamination level; if there is excessive foaming as a result, we can handle that as well. The carbon source or electron donor stock is useful during this process to add as a bolus to bulk up the culture again before switching back to continuous culture. Obviously after treatment in this way, the waste pot should be changed and the contents destroyed appropriately so as to ensure antibiotic waste does not enter drains, in keeping with good antibiotic stewardship principles.

We also keep several spare $\mathrm{pH}$ electrodes (autoclaved in glass measuring cylinders with damp cotton at the bottom) to hand, since they frequently fail during long runs, and a spare Clark oxygen cell probe treated in the same way, though they usually fail on the first day, if at all.

\subsection{Analytical Chemistry}

Very good determinations of the carbon source or electron donor are needed for the chemostat to be of any valid use for kinetic purposes. A lot of kits from, e.g., SigmaAldrich are based on enzymatic reactions and are quite specific but offer microtiter plate methods - these should be avoided at all costs since tiny pipetting errors change the path length and $1 \mathrm{~mm}$ path length cuvettes are far superior for reproducible assays. In this section we summarize some of the methodologies we have found to be reliable.

It is absolutely critical that determinations are done using "Class A" volumetric glassware and glass pipettes (or very well-calibrated "Gilson" micropipettors) and with the precision one would anticipate in the analytical chemistry laboratory. External standards in the form of certified reference materials or standard solutions should always be used and should not be made by the user. These are separate from calibrants and should not be used to make them.

\subsubsection{Carbohydrates and Intermediates of Krebs' Cycle}

While high-performance liquid chromatography (HPLC) is useful as it can be automated, it is not well-suited to obtain regular data ad hoc owing to setup, 
calibration, time, etc., particularly on communal instrumentation. Colorimetric tests that are done stringently and with good calibration can be as precise - they can be calibrated against HPLC to understand their error margin. Carbohydrate and Krebs' cycle intermediate kits are available from Sigma-Aldrich, Megazyme, and many others, which are very reliable and economical (and can be made oneself for even less money), and they can be made even more so by using smaller reaction volumes, etc.

\subsubsection{Common Electron Donors}

Kelly and Wood (1994) outlined methodologies for determination of thiosulfate, trithionate, and tetrathionate in mixtures and a second for tetrathionate, pentathionate, and hexathionate - between them, they allow all of these oxyanions to be quantified. For elementary sulfur sols, sulfide, sulfite, and thiocyanate, Kelly and Wood (1998) curate methods, along with those for various volatiles - carbon disulfide, dimethylsulfide, etc.

\subsubsection{Phosphate}

For phosphate-limited chemostats (or to demonstrate phosphate is not limiting), we determine phosphate using malachite green phosphate methods as we have found them to be very reliable in the $0-100 \mathrm{mM}$ range - they are interfered with by arsenite so for arsenite-limited chemostats, chromatographic separation may be needed. Many commercial kits are sold - we have found the one from R\&D Systems (Abingdon, UK) to be very satisfactory.

\subsubsection{Nitrogen Sources}

The phenol hypochlorite method of Solórzano (1969) is probably the most precise colorimetric method for ammonia determination. Nitrate and nitrite methods as given by Kelly and Wood (1998) are the ones that we use.

\subsection{Automatic Titration}

Most parameters of the chemostat are controlled by a microprocessor control unit, with various elaborate systems that $\log$ all parameters every $5 \mathrm{~s}$ and report them in a spreadsheet, etc. now available.

Very useful testing devices can be obtained from, e.g., Electrolab Biotech (Tewksbury, UK) which comprise resistors that are connected to probe ports on the controller and should give a reading of, e.g., $\mathrm{pH} 7.00$ - these make it possible to determine cable, controller, and probe faults with rapidity. Variable devices with multiple resistors in parallel can "fake" every $\mathrm{pH}$ unit or $\mathrm{dO}_{2}$ concentration.

\subsubsection{Temperature}

A good thermocouple probe is necessary, and while they do not need calibration, they should be checked periodically in baths of water of known temperature so that the user is aware of "drift." For workers with thermophiles, specialist probes may be 
needed versus the $<50{ }^{\circ} \mathrm{C}$ probes usually sold with most systems. Temperature can be controlled with a heated probe or a heated jacket - we have come to prefer the latter - oil, water, or electrically heated options are possible; the latter is much more costly, but it makes it easier to see the culture as the jacket can be removed very quickly.

\subsubsection{Oxygenation}

Clark oxygen cell probes require a lot more care than they are often given - the terminal membranes need cleaning in enzymatic solutions periodically, and the electrolyte needs replacement after every run. Such probes are calibrated after autoclaving by purging the system (basal medium and substrate already in the vessel) with oxygen-free nitrogen for at least an hour at $1 \mathrm{~L} / \mathrm{min}$ to give a base line " $\mathrm{dO}_{2}=0 \%$ " value to the control unit. It is then purged with laboratory air for a further $1-2 \mathrm{~h}$ to give a " $\mathrm{dO}_{2}=100 \%$ " value to the control unit. A properly calibrated probe is essential for determination of, e.g., $K_{\mathrm{L}} a$ during a run but can also be a valuable tool for determining reaction stoichiometry and for checking culture health - if the air supply and stirring are turned off and a bolus of carbon source added, the $\mathrm{dO}_{2}$ should fall - if it doesn't, the culture may be dead. A similar test is done at each steady state - at, e.g., a C-limited steady state, a bolus of medium containing vitamins, metals, nitrogen, and phosphate is injected - if the $\mathrm{dO}_{2}$ falls and then the amount of biomass increases, the culture was not limited by carbon. Specialist enzyme-based cleaners are available for the membranes on Clark oxygen cell probes, but we have used "protein remover tablets" for hard contact lenses with equal effect!

Pirt (1975) gives a lot of detail re: modes of oxygenation, and the reader should consult Pirt for any complex questions re: oxygenation - Pirt gives a lot of detail on stirring rates, headspace volumes, and gas sparging as means of oxygenation. As a basic rule, the air sparge should be at the bottom of the vessel, directly under the impellor. We use two Rushton pattern impellers, one directly above the other with the blades out of step, positioned directly over the sparge arm. In our laboratory for a c. $500 \mathrm{~mL}$ culture volume, we use $2-4 \mathrm{~L} / \mathrm{min}$ of laboratory air sparged into the bottom of the vessel through a punctured blind-ended tube to maintain $\mathrm{dO}_{2}$ at $50.0 \%$ for autotrophs on nonvolatile substrates - heterotrophs need less as they are not dependent on the $0.04 \%(v / v)$ carbon dioxide but the $21 \%(v / v)$ molecular oxygen. If working with, e.g., carbon disulfide, we would use $1 \mathrm{~L} / \mathrm{min}$ to avoid pushing the electron donor out of the medium before it can be consumed. In the Scott Laboratory at the University of South Florida (Tampa, FL), where Thiomicrospira, Thiomicrorhabdus, and Hydrogenovibrio spp. are cultivated at very low $\mathrm{dO}_{2}$, this is achieved by sparging briefly with a pulse of pure molecular oxygen, followed by rapid stirring. The stirring then stops, and at intervals, pure molecular oxygen pulses, each followed by brief stirring, are provided - this keeps the $\mathrm{dO}_{2}$ very low in a highly reproducible manner (Kathleen M. Scott, personal communication).

A common issue when growing organisms on gaseous carbon sources (e.g., methane), or when growing obligate autotrophs that will be attracted to the carbon dioxide in the air feed, is biofilm formation in the holes of the sparge arm, which can eventually block it. While this can sometimes be rescued by turning the air up to a 
high velocity, more often than not, this fails - those biofilms are surprisingly strong! We have found that a Bunsen valve over the sparge arm will prevent this - a piece of silicone tubing is slid over the arm and tightly attached with cable ties and each end. A razor blade is then used to slit a wiggly line that weaves in and out between the sparge holes. During use, this creates two flaps that will flap open and closed continually as gas enters the reactor, completely preventing biofilms from taking hold and blocking gas ingress even in runs of $>1$ year in length.

\subsubsection{Proton Concentration}

Proton concentration is usually monitored using a gel-filled (i.e., autoclavable) combination $\mathrm{pH}$ electrode. This is calibrated before the system is autoclaved,

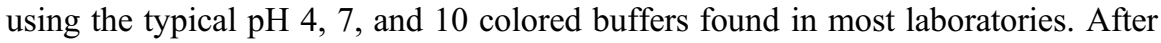
calibration, it should be checked with a $\mathrm{pH} 5$ or $\mathrm{pH} 8$ buffer not used for calibration to ensure it is working correctly. It is autoclaved in the vessel, usually with medium present to prevent damage to the probe. After autoclaving, the system is cooled completely before connecting the probe to the control unit. We have found it critical to have two to three spare probes to hand which can be calibrated and then autoclaved in measuring cylinders in about $1 \mathrm{~h}$ to rescue the system if the probe starts to malfunction - biofilm growth on the probe junction is the most common reason for this, which cannot be prevented, but is usually so minimal that it does not disrupt the steady state. During routine daily monitoring of the system, samples can be removed for external $\mathrm{pH}$ determination using a $\mathrm{pH}$ electrode or by titration, and in most modern systems, these data can be used to correct a drifting $\mathrm{pH}$ electrode very easily but are also useful data.

The microprocessor control unit controls two pumps - one that adds acid and one for base - that respond to fluctuations in $\mathrm{pH}$. Typically the acid and base are matched to the medium, e.g., if using thiosulfate as an electron donor for which the end product of growth is sulfate, sulfuric acid is usually used. We typically use $1 \mathrm{~N}$ acid and base for $\mathrm{pH}$ control - larger reactors, however, need stronger solutions to avoid excess dilution of the culture. For growth of autotrophs under electron donor limitation, the base used is commonly potassium carbonate since this ensures dissolved inorganic carbon is not the limiting substrate. The tubing from the acid and base reservoirs should be very long and moved along every week as the pump tends to "chew" the tubing resulting in non-addition or leaks. We have found the surgical steel used to manufacture the "triple addition" ports on many systems is prone to acid attack and greasing it with high-vacuum grease tends to minimize this, but having a spare, sterilized port on hand is important in these types of reactor as it is a common point of failure! Using a weaker acid is an option but does dilute the culture excessively.

Where precise determination of acid/base volume addition is needed (which is the case when any kinetic parameters are to be calculated), two options are used - one is an autotitrator that records the distance the pump has traveled; thus from tubing of fixed caliber, it calculates how much acid/base has been added since last reset. Alternatively, $500 \mathrm{~mL}$ or $1000 \mathrm{~mL}$ Class B burettes are readily available for about US\$260-300 and can simply be used as acid/base reservoirs with the tap left open 
and cotton wool stuffed into the neck. We have used both options, and providing there is an autoclave big enough for these burettes, they are a good option that is more economical in the long run and perhaps less prone to error. They are simply topped up during use and their volume recorded during daily monitoring.

\subsection{Starting, Running, and Finishing}

\subsubsection{Starting a Run}

The beginning of a run is commenced immediately after polarization of the oxygen probe. The medium in the vessel is fully aerated at this stage, and a late-exponential phase batch culture of about $10 \%(v / v)$ of the culture volume is added to the vessel. This can be done via addition ports from an inverted universal bottle or by injection through a septum port - the latter can kill a lot of cells if done too rapidly, however. For a nonvolatile, nongaseous substrate, the culture is aerated and sparged without any control (i.e., without attempting to keep $\mathrm{dO}_{2}$ at $50 \%$ ), but the $\mathrm{pH}$ is monitored and controlled. Usually we then run as a standard batch culture for $24-48 \mathrm{~h}$ until we are confident the amount of biomass is rising and that acid or base has been consumed by the culture. At this stage, we begin to move to a steady state as described later in this section. For volatile substrates, after inoculating the fully aerated, substrate-free medium, the air sparging is turned off, and the stirring is reduced to $50 \mathrm{rpm}$ to just keep the culture moving. Substrate is injected into the vessel, and the batch culture phase is continued without adding any addition of air. How much substrate is added at this stage is determined as follows:

If the culture is of volume $V$ and the vessel is of volume $L$, the headspace volume $H$ is determined by subtracting $V$ from $L$. Assuming laboratory air to be about $20 \%$ molecular oxygen, the volume of oxygen is $0.2 \mathrm{H}$ at the point of inoculation. The stoichiometry of total oxidation of substrate to carbon dioxide, water, etc. (i.e., the full oxidation as if all of the substrate was being used for energy and not for biomass, thus the hypothetical maximum amount of oxygen required) is determined - for example, for benzene:

$$
\mathrm{C}_{6} \mathrm{H}_{6}+7 \frac{1}{2} \mathrm{O}_{2} \rightarrow 6 \mathrm{CO}_{2}+3 \mathrm{H}_{2} \mathrm{O}
$$

The amount of molecular oxygen in the headspace is determined from $0.2 H / 22.4$, for example, in a vessel where $L=6 \mathrm{~L}, V=4 \mathrm{~L}$, and $H=2 \mathrm{~L}$; thus there is $17.8 \mathrm{mmol}_{2}$ present; given this, the stoichiometry can be adjusted to:

$$
2.4 \mathrm{C}_{6} \mathrm{H}_{6}+17.8 \mathrm{O}_{2} \rightarrow 14.2 \mathrm{CO}_{2}+7.2 \mathrm{H}_{2} \mathrm{O}
$$

Thus it is determined that $2.4 \mathrm{mmol}$ benzene is the maximum that can be utilized for the given amount of headspace molecular oxygen, and this amount $(165 \mu \mathrm{L})$ is injected into the vessel, giving a final concentration of $0.6 \mathrm{mM}$. Obviously, sensu stricto, one should also take into account the dissolved oxygen in the medium; however, the purpose of this step is merely to avoid "overfeeding" the culture and 
risking killing it but give it enough substrate to grow well given the amount of oxygen present - so we ignore the relatively small fraction of oxygen dissolved in the medium. The concentration of benzene is then monitored over $24-48 \mathrm{~h}$ (or longer), and once totally or almost totally consumed, the culture is sparged with air for $1 \mathrm{~h}$ to fully aerate it, and a further $2.4 \mathrm{mmol}$ is injected. Once this process has been repeated three times, the culture is usually sufficiently "bulked up" and "hungry" to begin conversion to continuous flow as described later in this section.

For gaseous substrates it is usual to mix them into the air supply - for example, methane is typically added at $30 \mathrm{~mL} / \mathrm{min}$ to $2 \mathrm{~L} / \mathrm{min}$ air - it is critical with flammable gases to ensure that the mixture produced is outside of the explosive limits (for methane, in the above example, it is $1.5 \%(v / v)$ in air, where the explosive range is $5-15 \%$ in air). This is simply sparged through the culture, and it is to all intents and purposes treated like a nonvolatile substrate, as described above. For most methanotrophs, carbon dioxide would also be added at about $5 \mathrm{~mL} / \mathrm{min}$ for the first $24-48 \mathrm{~h}$ - it is not entirely known why this is required, but it is the case that most of them require it to kick-start growth in some way.

The conversion to continuous flow is best done slowly. We make use of a "timer plug" of the ilk used to switch on lights when on holiday at set intervals, with a peristaltic pump connected. This allows flow to be continued for blocks of time without any hands-on work. We usually begin by determining a low dilution rate - around $D=0.01 \mathrm{~h}^{-1}$ or so - sometimes lower. In the tubing running from the medium reservoir to the vessel, we have a " $T$ " connector to which the "stem" of the " $T$ " is connected to a $10 \mathrm{~mL}$ analytical quality graduated pipette or burette via a short length of tubing that can be clamped. The top of the pipette has another length of tubing connected to a filter. This enables the flow rate $(F)$ of the medium to be determined. The pump is positioned between the "T"-piece and the vessel. Briefly, one clamps the tube close to the pump then attaches a syringe to the filter and draws up medium from the reservoir into the pipette. The tubing between the " $T$ " and the reservoir is then clamped and the pump turned on at various speeds, and triplicate determinations of $F$ are made by timing how long it takes to pump $5 \mathrm{~mL}$ out of the pipette and dividing down to give $F$ in $\mathrm{mL} / \mathrm{min}$. If we then want $D=0.01 \mathrm{~h}^{-1}$ initially and we have a $2000 \mathrm{~mL}$ culture volume (unlikely ours is actually $492.5 \mathrm{~mL}$ and precise volume must be known with all probes in place!), we can then ascertain (since $D=F / V$ ) that we need $F$ at $20 \mathrm{~mL} / \mathrm{h}$, or $0.33 \mathrm{~mL} / \mathrm{min}$, and the pump set appropriately. We then have the pump running as "1 hour on, 1 hour off" for about $12 \mathrm{~h}$ or so and then " 3 hours on, 1 hour off" for $12 \mathrm{~h}$, and after that, there is usually enough biomass to support running the pump continually - if the culture washes out completely, reinoculate and repeat but run " 3 hours on, 1 hour off" for 1-2 days before going continuous. It is worth noting that some models of reactor (e.g., Fermac models from Electrolab Biotech Ltd) have a "medium" pump built in that is set to deliver $x \mathrm{~mL} / \mathrm{min}$ by running at one speed for short lengths of time and then being off thus the medium is being added in pulses - this must be avoided as it will not give any useful data. We find it useful to ensure the tubing from reservoir to vessel has 3-4 lengths of tubing of different caliber in series so that we can work at very low and very high $F$ by moving the position relative to the pump. 


\subsubsection{Steady State}

Once the culture has been running at a fixed $D$ for several days, the amount of biomass $(x)$ will start to stabilize, and the concentration of the limiting substrate will deplete to $0 \mathrm{M}$ (thus the change in the amount of limiting substrate $(\Delta S)$ is the same as the initial concentration thereof) - at this stage, the yield $(Y)$ should be calculated every time the amount of biomass is monitored - on the basis of all units being converted to "per liter" rather than "per chemostat volume":

$$
\boldsymbol{Y}=\Delta \boldsymbol{x} / \Delta \boldsymbol{S}
$$

The unit of $Y$ can be $\mathrm{g}$ dry biomass/mol electron donor, g dry biomass/mol glucose, g dry biomass/mol glucose carbon, etc. - we tend to use all of them, depending on the precise context. A steady state is achieved when $Y$ stabilizes, not $X$ - the substrate consumed must be considered. We have a " $\mathrm{T}$ " connector in the tube just after the medium reservoir with a vaccine stopper on the "stem" of the "T" so we can withdraw sterile medium and monitor it for the precise substrate concentration over time - this is important as many substrates will oxidize to some degree in air. Once $Y$ is stable, $5 V$ of medium is passed through the vessel before a steady state is stable and can be made use of - some workers use $3 \mathrm{~V}$, but we have found in our hands that $5 V$ is more reliable, even if it does consume much more medium. At this point, replicate determinations of $Y$ are made. $D$ is similarly determined based on both $F$ and the volume per hour of acid/base being consumed since they add to the dilution of the culture - they are added to $F$ to give the true flow rate, and thus $D$ is determined. For kinetic studies, about ten steady states at a range of $D$ are determined by increasing/decreasing flow - we usually start low and speed up - and from these data, various useful parameters can be determined as discussed later in this chapter.

If removing samples from the chemostat at steady state for other work, a maximum of $0.1 V$ should be removed per day (Pirt 1975). We find it more convenient to connect a small $(2 \mathrm{~L})$ sterile waste container buried in wet ice and to collect overflow when we need larger amounts of biomass for work. For thermophiles, putting this bottle in cold water is enough to cool the overflow. From a $D=0.15 \mathrm{~h}^{-1}$ run of our $c .500 \mathrm{~mL}$ culture volume system, we can obtain $1.2 \mathrm{~L}$ of overflow in a 16-h overnight collection ready for work the next day.

At steady state, contamination checks must be carried out daily. In our laboratory we mostly work on obligate autotrophs, and our checks would be to streak out onto, e.g., EBS agar containing thiosulfate on which the autotroph will grow and Reasoner's 2A agar (Reasoner and Geldreich 1985) on which heterotrophic contaminants will grow. We also produce and examine Gram-stained slides daily and keep these for the duration of the run so that we can go back and check for any low-level contamination retrospectively. In a log book, we note temperature, $\mathrm{pH}, \mathrm{dO}_{2}, E_{h}$, volume of acid and base added thus far, medium flow rate (it should be checked periodically as pumps can "slip" during long runs), optical density (thus the amount of biomass), and substrate concentration in the reservoir and the vessel. While we 
use a computer to automatically log data from the $\mathrm{pH}$ probe directly, it is still essential to log manually lest many months of data are lost if the software crashes.

\subsubsection{Washout Kinetics: $\boldsymbol{\mu}_{\text {MAX }}$}

The parameter of critical dilution rate $\left(D_{\text {crit }}\right)$ is that at which the culture begins to washout rather than increase in $Y$. It can be hard to determine directly since when a culture is growing at $D \geq 0.9 D_{\text {crit }}$, erratic behavior and a fall in $Y$ are not uncommon (Pirt 1975), so while $D_{\text {crit }}$ is hypothetically equal to the maximum specific growth rate of the organism $\left(\mu_{\mathrm{MAX}}\right)$, it is hard to determine $D_{\text {crit }}$ simply by trial and improvement until a washout is observed: it is best determined from washout kinetics. We do this at the end of a run, usually, and it can be done within a working day. We use the method of Karagouni and Slater (1978), in which firstly a steady state is established at any $D$ at which the culture is stable but is reasonably high, so that $Y$ is also high enough that falls are easy to measure. Based on the specific growth rate $(\mu)$ from shake-flask batch culture on the same substrate, the $\mu_{\mathrm{MAX}}$ is estimated to be roughly in the same region - thus if working at $D=0.08 \mathrm{~h}^{-1}$ and shake-flask culture gave $\mu=0.12 \mathrm{~h}^{-1}$, for the purposes of the next step, we make the assumption that this is somewhere around $\mu_{\mathrm{MAX}} . D$ is then increased to, e.g., $0.20 \mathrm{~h}^{-1}$ so that it is well above the estimated $\mu_{\mathrm{MAX}}$, and $Y$ is determined every hour for 5-10 h. By plotting the decrease in $Y$ against $D$, it is possible to determine the actual, transient $\mu$ of the organism at each point of the washout by regression. Further increasing $D$ allows this to be determined several times - this could continue until the culture washes out to the point at which one cannot determine $Y$ any more. From these data, it is possible to determine the highest $\mu$ evident during washout, which is the true $\mu_{\mathrm{MAX}}$.

An alternative method for the determination of $\mu_{\mathrm{MAX}}$ is that of Pirt and Callow (1960), in which we establish a steady state at a moderate $D$ and determine $x$ there (which we call $x_{\mathrm{i}}$ ). $D$ is then increased to a much higher value, and $x$ is determined at intervals over 5-10 h. A plot of $\ln x$ versus time $(t)$ is then produced, for which:

$$
\ln \boldsymbol{x}=\left(\boldsymbol{\mu}_{\mathrm{MAX}}-\boldsymbol{D}\right) \boldsymbol{t}+\ln \boldsymbol{x}_{\mathbf{i}}
$$

Thus, the gradient of the line is $\mu_{\mathrm{MAX}}-D$, and since the latter is known, the former can be calculated easily.

\section{Determination of Parameters}

We have already given the determination of $\mu_{\mathrm{MAX}}$ in Sect. 3.5.3, since it is determined experimentally rather than from the examination of data from many experiments. In this section, we consider the determination of other parameters from chemostat yield data. 


\subsection{Maximum Specific Molar Growth Yield $\left(Y_{\text {MAX }}\right)$ and Maintenance Coefficient $\left(m_{s}\right)$}

The maximum specific molar growth yield ( $\left.Y_{\mathrm{MAX}}\right)$ is known by many names maximum yield coefficient, theoretical yield, true yield, and so on. To understand what this coefficient means and how it is determined, it is best considered alongside the maintenance coefficient $\left(m_{\mathrm{S}}\right)$.

The concept of what we refer to as "Pirtian maintenance," sensu the maintenance coefficient $\left(m_{\mathrm{S}}\right)$ has been argued against by many authors over the years, and the validity of the concept and the methods of determination have both come under scrutiny - von Bodegom (2007) gives an excellent critical evaluation of the concept. It is important the note that $m_{\mathrm{S}}$ in the Pirtian sense is not the only interpretation of or means to evaluate maintenance as an overall concept, and thus while some shun this particular parameter in terms of others, it still has purpose - should the reader wish to explore the full gamut of maintenance parameters, von Bodegom's (2007) review is an excellent springboard. Pirt (1965) gives a good overview of the derivations and significance of Pirtian maintenance parameters and, along with Pirt (1975), provides a very useful canon of Pirtian kinetic theory.

Pirt stated that a fraction of the total electron donor consumed $\left(\Delta S_{\mathrm{E}}\right)$ was used in part for growth $\left(\Delta S_{\mathrm{G}}\right)$ and in part for maintenance $\left(\Delta S_{\mathrm{M}}\right)$, the latter being for repair and replacement of damaged cell components. Since $\Delta S_{\mathrm{M}}$ is probably always $>0$, it is impossible to determine a true specific molar growth yield in the sense of just growth; since the $\Delta S$ component of the parameter (cf. Sect. 3.5.2) is total substrate ( $\Delta S_{\mathrm{E}}$, if an electron donor/energy source) consumed, $Y$ is effectively "amount of biomass formed per unit substrate consumed, regardless of what for." If it were possible to view $\Delta S_{\mathrm{M}}=0$, we would see the "true" growth yield $Y_{\mathrm{MAX}}$ :

$$
\boldsymbol{Y}_{\mathbf{M A X}}=\Delta \boldsymbol{x} / \Delta \boldsymbol{S}_{\mathrm{G}}
$$

$Y_{\text {MAX }}$ is the highest possible yield parameter for growth per unit electron donor, since no substrate is consumed for maintenance - obviously this cannot be determined directly experimentally. $Y_{\mathrm{MAX}}$ is instead determined from $Y$ at a range of $\mu$ :

$$
1 / Y=m_{\mathbf{S}}(1 / \mu)+\left(1 / Y_{\text {MAX }}\right)
$$

After determining $Y$ at a range of steady states each at a different $\mu$ (i.e., $D$ ), $Y_{\mathrm{MAX}}$ can be determined from the above relationship. Historically, this was done using graphical methods akin to the Lineweaver-Burk plot (Lineweaver and Burk 1934) in enzymology (compare $1 / V$ vs. $1 / S$ and $1 / Y$ vs. $1 / \mu$ ), but they are highly error laden, and hyperbolic fitting is now so much easier to undertake. Rather than explain at length how to go about doing it, there are many YouTube resources explaining how to determine $V_{\max }$ from enzymology data using hyperbolic fitting, for example, using the "SOLVER" add-on for Microsoft Excel, which reduces the task to a few minutes work - these should be consulted if the reader is not familiar with reducing the sum of squared residuals, etc. - alternative methods using R, Python, etc., are well-suited 
to batch processing of datasets or for particularly large datasets. Hyperbolic fitting, if done following the Michaelis-Menten methodologies, will not give $m_{\mathrm{S}}$ directly, but via a second parameter, the specific maintenance rate $(a)$, which can be considered as a turnover rate for biomass - on its own it is not particularly useful, but it can be used to determine $m_{\mathrm{S}}$ since:

$$
m_{\mathrm{S}}=\boldsymbol{a} / \boldsymbol{Y}_{\mathrm{MAX}}
$$

Again, historically $m_{\mathrm{S}}$ was determined graphically, usually from plots of the metabolic quotient $(q)$ versus $\mu$. Since:

$$
\boldsymbol{q}=\boldsymbol{\mu} / \boldsymbol{Y}
$$

this plot is analogous to the Hanes-Woolf plot from enzymology (Hanes 1932) and thus is prone to the same errors (Haldane 1957). The metabolic quotient $q$ is sometimes termed the specific rate of substrate uptake or the specific metabolic quotient; it is often written in terms of the limiting substrate $-q_{\text {thiosulfate }}, q_{\text {glucose }}$, etc. - if the limiting substrate is respiratory molecular oxygen, $q_{\mathrm{O} 2}$ is properly termed the "respiration rate." The same could be said of any anaerobic terminal electron acceptor, though while $q_{\mathrm{NO} 3-}$ is a respiration rate, $q_{\mathrm{O} 2}$ is the respiration rate! $q$ is usually given in mmol substrate per gram dry biomass - we consider these parameters in Sect. 4.2.

The maintenance coefficient $m_{\mathrm{S}}$ is usually given in g electron donor per g dry biomass.h and is a measure of how much of the energy source is required to maintain a gram of dry biomass for an hour. It can be converted to $m_{\mathrm{ATP}}$, which is given in mmol ATP per g dry biomass.h - the amount of ATP required to maintain a gram of dry biomass for an hour - though this conversion requires understanding of the production rates of ATP per unit electron donor. This can be easily determined in resting cell suspensions held in a thermostatted vessel at the usual growth temperature (we use the chamber of a Rank Brothers Ltd (Bottisham, Cambridge, UK) Clark-type oxygen cell along with a recirculating water bath as an easy option, but small flasks in a water bath may suffice, though they will take longer for the contents to reach temperature). To these cells, a known amount of pre-warmed electron donor is added, and aliquots are then removed at 10 -s intervals into $10 \%$ of the aliquot volume of $1.45 \mathrm{M}$ perchloric acid containing $10 \mu \mathrm{M}$ xylenol blue (Cohen 1922) and are held on ice for at least 10 min for digestion to complete. This all must be carried out in hypochlorite-washed, baked glass containers to ensure background ATP is fully removed. After incubation on ice, $1.00 \mathrm{~mL} 0.3 \mathrm{M}$ potassium hydroxide is added per $0.2 \mathrm{~mL}$ perchloric acid solution used in the digestion. This brings about neutralization, as indicated by the indicator changing from red/yellow to just violet - if it is not neutralized, further alkali should be added. The heavy white precipitate formed is allowed to settle and the concentration of ATP is determined in the decanted supernate using commercial firefly luciferase kits and a luminometer (though x-ray film or a scintillation counter can also be used for light detection - cf. Boden et al. 2010) - it is important that the ATP calibrants are subjected to perchloric acid and 
potassium hydroxide since potassium perchlorate does afford some quenching of the luminescence (Kelly 1965; Boden et al. 2010). The final cell suspension left in the incubation chamber is rapidly filtered to remove biomass, and the filtrate is assayed to determine the concentration of the electron donor - from these data, one can determine both the ATP production rate versus a control incubated without electron donor and the amount of ATP formed per mol electron donor - the latter allowing determination of $m_{\text {ATP }}$ (the methods we summarize here are from Boden et al. 2010, 2011b; Kelly 1965; Kelly and Syrett 1966; Hutt 2016 - the reader should consult these texts for full technical detail and example datasets).

$m_{\mathrm{S}}$ and $m_{\mathrm{ATP}}$ are measures of the "cost of living" of the organism - if a nonhalophile is subjected to salinity, for example, the amount of biomass formed $(x)$ decreases, but the amount of electron donor consumed stays the same or can increase - and compared to growth without salt stress, there is more electron donor consumed per unit biomass (since the latter has decreased); this additional consumption is owing to a rise in $m_{\mathrm{ATP}}$, thus $m_{\mathrm{S}}$; the organism demands more electron donor turnover to simply stay alive. As to what maintenance is comprised of, we know from the work of Stouthamer and Bettenhausen (1973) and Watson (1970) on solute stress in Bacteria and unicellular Eukarya that maintaining osmotic balance and gradients requires a significant fraction of the maintenance energy. Studies by Harrison and Loveless (1971) working at or below the optimum temperature of Escherichia coli showed no increase in $m_{\mathrm{S}}$ with temperature change; thus they (and later Pirt (1975)) assumed that $m_{\mathrm{S}}$ was not effected by temperature - though since they were not working at temperatures likely to cause significant damage (i.e., toward the very upper limits of growth), there is no reason why $m_{\mathrm{S}}$ would increase at the temperatures examined. Maintaining proton gradients and repairing damage induced by $\mathrm{pH}$ changes resulted in a reasonably high increase in $m_{\text {ATP }}$ (Harrison and Loveless 1971), indicating that this is a major use of maintenance energy.

One of us studied the effects of $\mathrm{Hg}(\mathrm{II})$ ions on Methylococcus capsulatus bath in cell suspensions using $\left[{ }^{14} \mathrm{C}\right]$-methane as a tracer (Boden and Murrell 2011). In the absence of $\mathrm{Hg}(\mathrm{II})$, the partitioning of methane-carbon between biomass and carbon dioxide was $61 \pm 4 \%$ assimilated to biomass and $23 \pm 3 \%$ dissimilated to carbon dioxide over $1 \mathrm{~h}$, with the remainder presumed to be as soluble intermediates. When $10 \mathrm{mM} \mathrm{Hg}$ (II) was added, all of the methane was very rapidly oxidized to carbon dioxide within $30 \mathrm{~min}$, and absolutely none was assimilated into biomass. These data demonstrate a very sharp rise in the maintenance cost of the organism - when $\mathrm{Hg}$ (II) is present, the constitutively expressed mercuric reductase (EC 1.16.1.1) reduces the ion to elementary mercury to detoxify it, requiring 1 mol NADH per mol $\mathrm{Hg}$ (II) detoxified - in this case, $0.5 \mathrm{mmol} \mathrm{NADH}$ would be needed by the $50 \mathrm{~mL}$ culture. As the flasks contained $9 \mathrm{~mL}$ methane $(0.4 \mathrm{mmol})$, and we know that methane dissimilation in this organism using the particulate methane monooxygenase (EC 1.14.18.3) generates a net 1 mol NADH per mol methane fully dissimilated (Anthony 1982), it is easy to see that during that 30 -min period, about $80 \%$ of the mercury would have been detoxified owing to this rapid oxidation of the electron donor purely for energy. In cases like these where a bolus of electron donor is rapidly "burnt" to yield energy for a specific detoxification - be that of metal ions or of 
protons or of a toxic organic - the maintenance coefficient of the organism must rise such that $\Delta S_{\mathbf{M}}=\Delta S$; the amount of electron donor required to maintain the organism has become equal to the total amount of electron donor consumed, as absolutely none is being used for growth. Such situations are impossible to monitor in the chemostat since if no growth occurs, no change in $Y$ can be measured, of course - thus they must be derived as we have here from batch culture work and use of sensitive tracers - carbon-14 detection allows use at $\mathrm{fM}$ concentrations whereas carbon-13 tracing is often limiting and requires use at, e.g., 1-5\% of the total carbon source; so for a $10 \mathrm{mM}$ glucose culture, there would be $0.1-0.5 \mathrm{mM}$ carbon- 13 present. A thorough (if dated) review of radiotracers in cell physiology can be found in Aronoff (1957), which gives useful technical detail missing from later works.

A very elegant example of the use of $Y_{\text {MAX }}$ in physiology is that of Wood and Kelly (1986), who made use of comparison of $Y_{\text {MAX }}$ and a "corrected" version that accounted only for electron donor oxidized for ATP synthesis. This was done during aerobic growth of the obligate autotroph Thermithiobacillus tepidarius on a range of homologous electron donors - the polythionates, viz., trithionate, tetrathionate, hexathionate, and heptathionate - which increase by one interior sulfane sulfur at each step in the series (pentathionate was not used owing to complications of synthesis at the time). In theory, the ratio of "corrected" yields between adjacent polythionates in the chain will be consistently 7:4 if only oxidative phosphorylation contributes to ATP synthesis but would skew as chain length increased if substratelevel phosphorylation also contributed - this allowed a very elegant determination that only oxidative phosphorylation contributed to ATP synthesis in this organism. Similarly, $Y_{\mathrm{O}}$ (cf. Sect. 4.2) was fairly consistent between polythionates examined, suggesting that molecular oxygen as a terminal electron acceptor is the only route of ATP synthesis in this organism. The "corrected" $Y$ referred to here is specifically $Y_{\mathrm{G}}$ and is determined from:

$$
Y_{\mathbf{G}}=\Delta \boldsymbol{x} / \Delta S_{\mathbf{G}}
$$

which is the same means of determination of $Y$ but with consideration only of the amount of electron donor consumed for growth and not for maintenance.

\subsection{Metabolic Quotients $(q)$ and the Respiration Rate $\left(q_{02}\right)$}

We have covered in Sect. 4.1 the means of determination of $q$ and of what $q$ and $q_{\mathrm{O} 2}$ are in basic terms, but herein we cover their use.

The respiration rate $\left(q_{\mathrm{O} 2}\right)$ can be determined from the maximum respiration rate $\left(q_{\mathrm{O} 2 \mathrm{MAX}}\right)$ in oxygen-limited chemostats - the latter is determined from $q_{\mathrm{O} 2 \mathrm{MAX}}=\mu_{\mathrm{MAX}} / Y_{\mathrm{O}}^{\mathrm{MAX}}-$ the maximum specific molar growth rate with respect to oxygen and the maximum yield with oxygen as the limiting substrate:

$$
\boldsymbol{q}_{\mathrm{O}_{2}}=\boldsymbol{q}_{\mathrm{O}_{2} \mathrm{MAX}} \times \boldsymbol{p}_{\mathrm{O}_{2}} /\left(\boldsymbol{p}_{\mathrm{O}_{2}}+\boldsymbol{K}_{\mathbf{p}}\right)
$$


where $p_{\mathrm{O} 2}$ is the dissolved oxygen partial pressure (usually in $\mathrm{mmHg}$ ) and $K_{\mathrm{p}}$ is the Monod constant ( $K_{\mathrm{S}}$, cf. Sect. 4.4) for oxygen, but given in pressure units ( $\mathrm{mmHg}$ is the most commonly used unit in the literature, since most studies are in older texts - as long as the units used for $p$ and $K_{\mathrm{p}}$ are consistent, anything can be used - the Pascal $(\mathrm{Pa})$ is probably the most convenient, as the standard atmosphere $(\mathrm{atm})$ is not very convenient for dissolved gas partial pressures). If we then make the assumption that the specific molar growth yield per mole of oxygen $\left(Y_{\mathrm{O}}\right)$ is constant, we can change this to:

$$
\boldsymbol{\mu}=\boldsymbol{\mu}_{\mathbf{M A X}} \times \boldsymbol{p}_{\mathbf{O}_{2}} /\left(\boldsymbol{p}_{\mathbf{O}_{2}}+\boldsymbol{K}_{\mathbf{p}}\right)
$$

This gives the relationship between specific molar growth rate (per mole of oxygen in this case, $\mu$ ) and the concentration of dissolved oxygen as the limiting substrate. At a critical dissolved oxygen partial pressure $\left(p_{\text {crit }}\right), q_{\mathrm{O} 2}$ ceases to be coupled to $p_{\mathrm{O} 2}-p_{\text {crit }}$ is dependent entirely on $\mu$ under air at atmospheric pressure, but under hyperbaric conditions, the relationship between $q_{\mathrm{O} 2}$ and $p_{\mathrm{O} 2}$ becomes unpredictable and unstable - for further information on growth at elevated pressures, the reader should consult MacLennan et al. (1971), which uses Methylobacterium extorquens AM1 as a model organism during growth on methanol, or for relations between $p_{\text {crit }}$ and $\mu$ in general, Harrison et al. (1969).

\subsection{ATP Yield of Biomass $\left(Y_{\text {ATP }}\right)$}

The ATP yield of biomass $\left(Y_{\text {ATP }}\right)$ was originally defined (Bauchop and Elsden 1960) as:

$$
\boldsymbol{Y}_{\mathrm{ATP}}=M_{\mathrm{W}} \boldsymbol{Y}_{\mathbf{E}} / \boldsymbol{n}
$$

where $M_{\mathrm{W}}$ is the molecular weight of the electron donor (in Daltons), $n$ is the number of moles ATP produced by the metabolism of $1 \mathrm{~mol}$ electron donor, and $Y_{\mathrm{E}}$ is the specific molar growth yield expressed in $\mathrm{g}$ dry biomass per $\mathrm{g}$ electron donor. We would now of course simplify this to:

$$
\boldsymbol{Y}_{\mathrm{ATP}}=\boldsymbol{Y} / \boldsymbol{n}
$$

where $Y$ is expressed in $\mathrm{g}$ dry biomass per mole electron donor. We have already outlined how $n$ can be determined in Sect. 4.1.

The magnitude and meaning of $Y_{\mathrm{ATP}}$ have changed over the years since 1960, with it originally being thought to have a fixed value of about 10.5 , but this was later found by Stouthamer and Bettenhausen (1973) to be vastly underestimated. In various studies, Stouthamer estimated the maximum $Y_{\text {ATP }}$ to be 28.8-32.1 (Stouthamer 1973, 1976), but since chemostat kinetics has largely gone out of fashion in recent decades, the true gamut of $Y_{\mathrm{ATP}}$ and influencing factors has been largely neglected. 


\subsection{The Monod Constant $\left(K_{\mathrm{s}}\right)$}

The Monod constant $\left(K_{\mathrm{S}}\right)$ is also known as the saturation constant and is the equivalent of the Michaelis-Menten constant $\left(K_{\mathrm{M}}\right.$, Michaelis and Menten 1913) in as much as that it is the concentration of substrate at which $\mu$ is half of $\mu_{\mathrm{MAX}}$. It is termed the Monod constant after the "Monod relation" in which it appears (Monod 1942, 1949):

$$
\boldsymbol{\mu}=\boldsymbol{\mu}_{\mathrm{MAX}} \times \boldsymbol{S} /\left(\boldsymbol{S}+\boldsymbol{K}_{\mathbf{S}}\right)
$$

where $S$ is the concentration of the limiting substrate in the medium reservoir. An extension of the Monod relation by consideration of $Y$ at the same time gives the "Pirt equation":

$$
\boldsymbol{\mu}=\boldsymbol{\mu}_{\mathrm{MAX}} \times\left(\boldsymbol{S} /\left(\boldsymbol{S}+\boldsymbol{K}_{\mathbf{S}}\right)\right)-\boldsymbol{m}_{\mathbf{S}} \times \boldsymbol{Y}_{\mathbf{M A X}}
$$

The Monod constant has an inverse relationship with the affinity of an organism for its substrate - a high $K_{\mathrm{S}}$ refers to a low affinity:

$$
\mathbf{1} / \boldsymbol{\mu}=\left(\boldsymbol{K}_{\mathrm{S}} / \boldsymbol{S} \boldsymbol{\mu}_{\mathrm{MAX}}\right)+\left(\mathbf{1} / \boldsymbol{\mu}_{\mathrm{MAX}}\right)
$$

As such, hyperbolic fitting as outlined elsewhere in this chapter can be used to determine $K_{\mathrm{S}}$ from the specific growth rates in ( $\mathrm{pH}$ and oxygenation-controlled) batch culture at a range of substrate concentrations. Historically, this was done from a plot of $1 / \mu$ versus $1 / S$, in which the $x$-intercept is $-1 / K_{\mathrm{S}}$ and the $y$-intercept is $1 /$ $\mu_{\mathrm{MAX}}-$ such plots are still useful for visualizing data particularly when considering mixed metabolic modes (cf. Sect. 4.6). In spite of the relative ease of determination, $K_{\mathrm{S}}$ values are not widely reported in the literature - historically this was owing to understanding that the double-reciprocal plots gave high-error estimations for a largely very low parameter (Pirt 1975), though this has changed significantly in the twenty-first century with computational means of solving hyperbolic data. Most determinations for carbon sources are in the range of tens of $\mu \mathrm{M}$ - that for respiratory oxygen is near to $1 \mu \mathrm{M}$ and those for macronutrients such as magnesium and phosphate are similarly in the tens of $\mu \mathrm{M}$. Those for vitamins are as low as hundreds of pM (i.e., very high affinity), but trace metal values are seldom reported, and there is some evidence that the Monod relation breaks down at such low concentrations of a substrate (Pirt 1975).

\subsection{Oxygen Transfer Coefficient $\left(k_{\mathrm{L}} a\right)$}

Particularly important in biotechnology, the oxygen transfer coefficient $\left(k_{\mathrm{L}} a\right)$ is the proportionality constant for the rate of oxygen mass transfer into a liquid relative to the concentration gradient. $k_{\mathrm{L}} a$ can be determined through abiotic methods such as by the reaction of sulfite with dissolved oxygen to yield sulfate in the presence of 
$\mathrm{Cu}$ (II) or $\mathrm{Co}$ (II) ions as a catalyst (Cooper et al. 1944). The reactor is filled with $1 \mathrm{~N}$ sodium sulfite solution (freshly prepared!) containing $1 \mathrm{mM}$ cupric sulfate or cobaltous sulfate (it is prudent to add the sulfite solution to the reactor slowly and gently to avoid too much air mixing in, and to then add the $\mathrm{Cu}$ (II) or $\mathrm{Co}$ (II) stock solution and gently stir in using a glass rod), and then the air sparging is commenced, with a timer started the moment the first bubble leaves the sparge arm. At intervals, sparging is stopped, the solution stirred briefly, and an aliquot removed. Sulfite is determined in samples by adding excess standard iodine solution and titrating against thiosulfate, or by colorimetric means - both are given in Kelly and Wood (1998). From the determined sulfite concentrations, the rate of consumption $(v)$ can be determined (importantly not forgetting to convert from concentration to amount first - a common error!):

$$
2 k_{\mathrm{L}} a \times c_{\mathrm{S}}=-v
$$

where $c_{\mathrm{S}}$ is the solubility of oxygen in the liquid under test $\left(506 \mu \mathrm{M}\right.$ at $25^{\circ} \mathrm{C}$ in pure water - but this will vary in culture media, obviously). Pirt (1975) gives various methods for the determination of $k_{\mathrm{L}} a$ in cultures, and a detailed overview with an industrial process viewpoint can be found in Garcia-Ochoa and Gomez (2009).

\subsection{Comparing Metabolic Modes}

While we have noted above the errors in the $q$ versus $\mu$ and $1 / Y$ versus $1 / \mu$ plots, we do find them very useful for comparing growth conditions and in the determination of metabolic modes. In this regard, they can be used, e.g., akin to the inverse of the enzyme inhibitor methods of Cornish-Bowden (1974), when considering chemolithoheterotrophy or mixotrophy - we will come back to this later in this section. To do this, data are obtained by hyperbolic fitting and then replotted on the above axes, such that, e.g., the $y$-intercept on the $1 / Y$ versus $1 / \mu$ plot is the reciprocal of the $Y_{\mathrm{MAX}}$ value determined by hyperbolic fitting and not the natural $y$-intercept of the data points. This is often most useful when comparing data with older studies, and this can be done directly by reanalysis of the original work by using the WebPlotDigitizer tool (http://www.automeris.io), which has been demonstrated as reliable for extracting raw data from original plots and enables reevaluation (Drevon et al. 2016). Using this tool or equivalent, it is possible to extract raw data from every data point of $1 / Y$ versus $1 / \mu$ plots and to reanalyze them using hyperbolic fitting, which gives better determination of $Y_{\mathrm{MAX}}$ and $m_{\mathrm{S}}$ both directly and because in many $1 / Y$ versus $1 / \mu$ plots, best-fit lines were drawn in by hand and may not be the mathematical best fit of the dataset. To illustrate the value in these reanalyses, Fig. 2 shows data from chemostat cultures of Halothiobacillus neapolitanus NCIMB 11333 (Kelly and Syrett 1963) grown by Mason (1986) under electron donor limitation. Mason's original data are shown (points) with trend lines (black) based on her original $Y_{\mathrm{MAX}}$ determinations from graphical methods and our reanalysis (red) from extracted raw data reanalyzed using hyperbolic fitting. It can be seen that 


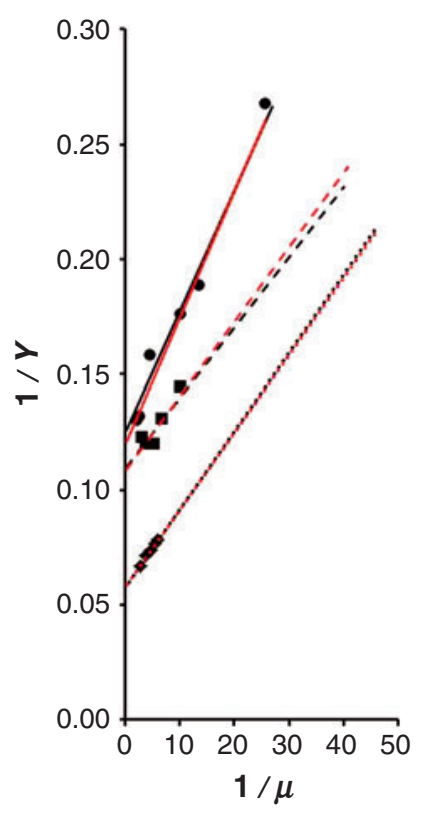

Fig. 2 Reconstructed $1 / D$ versus $1 / Y$ plot for Halothiobacillus neapolitanus NCIMB 11333 (Kelly's "Thiobacillus sp. C" (Kelly and Syrett (1963)) grown in electron donor-limited chemostats on thiosulfate (circles, solid trend lines), trithionate (squares, broken trend lines), and tetrathionate (lozenges, dotted trend lines) at a range of dilution rates by Mason (1986). Raw data were extracted from the same plot by Mason (1986) using the WebPlotDigitizer package (http://www.automeris.io) based on scanned images from the original and trend lines applied (black) based on Mason's $Y_{\text {MAX }}$ determinations (from $1 / D$ versus $1 / Y$ graphical methods) to set the $y$-intercept. Mason's raw data were reanalyzed by hyperbolic fitting using the SOLVER add-on for Microsoft Excel and the GRG nonlinear engine to reduce the sum of squared residuals. Newly determined $Y_{\mathrm{MAX}}$ and $m_{\mathrm{S}}$ data for each electron donor were used to construct new $1 / D$ versus $1 / Y$ series (red) by calculating $1 / Y$ values at a range of $D$ given the new $Y_{\mathrm{MAX}}$ and $m_{\mathrm{S}}$

there is very little difference between Mason's mid-1980s graphical determinations and our reanalyses with very little variance (new vs. old, g/mol) in $Y_{\text {MAX }}$ for tetrathionate (17.3 vs. 17.2), but more for trithionate (9.3 vs. 9.1) and thiosulfate ( 8.3 vs. 8.0 ). $m_{\mathrm{S}}$ was more variable (new vs. old, $\mathrm{mmol} / \mathrm{g} . \mathrm{h}$ ) but again less variable for tetrathionate ( 3.34 vs. 3.58$)$ than trithionate ( 3.23 vs. 3.04$)$ and thiosulfate (5.53 vs. 6.31), even though the original values are from $q$ versus $\mu$ plots. While these differences are small, they are important; thus it is good practice to reanalyze data where possible, and plots were made available by the original authors than to merely compare the coefficients produced with those from older studies.

There are a number of mixed metabolic modes that can be examined usefully using these graphical methods, such as with chemolithoheterotrophy (cf. Boden and Hutt chapter $\triangleright$ "Chemolithoheterotrophy - Means to Higher Growth Yields from This Widespread Metabolic Trait?" elsewhere in this handbook), where heterotrophic growth is supplemented by the oxidation of an auxiliary substance as an 
electron donor but there is no concomitant autotrophy. Endochemolithoheterotrophy is the variant in which an organism takes in, e.g., dimethylsulfide and grows on it heterotrophically (per Methylophaga spp.) and then endogenously generates a sulfide or thiosulfate moiety from the compound and in turn oxidizes that chemolithotrophically as an auxiliary substance (i.e., source of electrons), producing sulfate, thiosulfate, or tetrathionate as the end product - which has been observed in Methylophaga thiooxydans and other Methylophaga spp., as well as in some Hyphomicrobium spp. (Boden et al. 2010). Exochemolithoheterotrophy is the more commonly observed variety in which, for example, a Methylophaga sp. is grown heterotrophically on methanol and thiosulfate is supplied as an exogenous auxiliary substance, which is oxidized chemolithotrophically. Mixotrophy (often but wrongly - used as a blanket term for all or any mixed metabolic mode) occurs when an organism grows both heterotrophically, e.g., on glucose while growing simultaneously autotrophically at the expense of light or an auxiliary substance such as thiosulfate or formate, as is the case in Paracoccus versutus $\mathrm{A} 2{ }^{\mathrm{T}}$ (Wood and Kelly 1981). A further, more complex scenario occurs and has been observed in Xanthobacter tagetidis TagT2C ${ }^{\mathrm{T}}$ (Padden 1997; Padden et al. 1998) growing on substituted thiophenes in which carbon from the thiophene backbone is assimilated heterotrophically, while the sulfide moiety is oxidized chemolithotrophically as an electron donor. Some of the energy conserved from the latter oxidation supplements the heterotrophic growth as endochemolithoheterotrophy and some fuels the assimilation of carbon dioxide (some exogenous, some produced from the thiophene backbone) - thus we have mixotrophy in which the heterotrophic component is being supplemented with electrons from an auxiliary substance - endochemolithoheterotrophy in this case. This combination of mixotrophy in which the heterotrophy is chemolithoheterotrophy does not thus far have a name but we propose voracotrophy (Latin adj. vorax, voracious, inclined to devour; Gr. n. fem. trophê, food, nourishment), literally "feeding voraciously" or "feeding greedily" for this scenario in which an organism is literally using every metabolic mode that it has all at once. In this section, we will consider several common patterns observed in graphical representations of data and their equations, based on the Michaelis-Menten kinetic analogies for inhibitor studies.

\subsection{Analogous to Inverted Mixed Inhibition}

Returning to the H. neapolitanus data given in Fig. 2, these afford an example of using graphical methods to consider mixed metabolic modal kinetics using the inverse of common enzymology graphical methods. In Lineweaver-Burk plots, an uncompetitive inhibitor gives a parallel line to that of the uninhibited system, with a higher $1 / V_{\operatorname{MAX}}$, whereas a competitive inhibitor gives a nonparallel line that shares the same $1 / V_{\mathrm{MAX}}$ as uninhibited enzyme. A noncompetitive inhibitor gives a nonparallel line that has a different $1 / V_{\mathrm{MAX}}$ but converges at the same $x$-intercept as the uninhibited system, and a mixed inhibitor gives a nonparallel line that crosses the uninhibited system left of the ordinate. For growth stimulated by an auxiliary 
substance, obviously, the stimulated condition takes the place of the "uninhibited" line in enzymology, and the control condition takes the place of the "inhibited" line. So, if we consider the thiosulfate and trithionate data of Fig. 2, we must first consider the species - where thiosulfate is $\mathrm{S}_{2} \mathrm{O}_{3}{ }^{2-}$ and trithionate is $\mathrm{S}_{3} \mathrm{O}_{6}{ }^{2-}$, the latter behaves as $\left(\mathrm{S}_{2} \mathrm{O}_{3}{ }^{-}\right) \mathrm{SO}_{3}{ }^{-}$or $\mathrm{S}\left(\mathrm{SO}_{3}{ }^{-}\right)_{2}$ in terms of energetics and reactivity, particularly in cleavage reactions (Lyons and Nickless 1968) - since the substance is cleaved to thiosulfate and sulfite during metabolism by trithionate hydrolase (EC 3.12.1.1), thus we can consider it as the former: "thiosulfate plus sulfite," in which the latter is the auxiliary substance versus growth on thiosulfate alone. It should be noted that some reports on trithionate hydrolase suggest the sulfite moiety is immediately oxidized by the oxygen of water into sulfate - were this the case, $Y$ on trithionate and thiosulfate would be identical, since the only energetically active part of the oxyanion would be thiosulfate moiety, unless the protons released from water could be coupled to energy metabolism - the observation of the production of sulfate could simply be due to experimental issues owing the low stability of sulfite and tendency to oxidize immediately, and that is what we consider to be the case; it clearly warrants further study. One would expect a higher $Y_{\mathrm{MAX}}$ for trithionate versus thiosulfate if all three sulfurs were released from it in an oxidizible state (i.e., thiosulfate and sulfite), which is the case in Fig. 2. The trithionate line (broken) crosses that of thiosulfate (solid) somewhere left of the ordinate and that of trithionate will cross the abscissa much further left than that of thiosulfate: per a mixed inhibitor but with the lines inverted since it is stimulatory not inhibitory. Similarly, if plotted per an Eadie-Hofstee plot (Hofstee $1959-Y$ vs. $Y / \mu$ for chemostat data), nonparallel lines per the inverse of competitive inhibition (i.e., trithionate on top) are seen but with them crossing left of the ordinate (data not shown) - congruent with mixed inhibition. If we analogize with the inverse of the enzymological parameters of a mixed inhibitor, one would expect to see $m_{\mathrm{S}}$ decrease for growth on trithionate and see a decrease in the organism for the substrate - an increase in $k_{\mathrm{S}}$ for trithionate versus thiosulfate, both accompanied by an increase in $Y_{\mathrm{MAX}}$. In the same way as mixed inhibition works, sulfite (the "extra" part - acting as the inverse of an inhibitor) is metabolized via a different pathway to thiosulfate and thus is not bound by the same site - there is no competition. In terms of kinetic parameters, the extended Michaelis-Menten equation used for mixed inhibitor studies can be used to derive the inverse equivalent for Monod kinetics:

$$
\boldsymbol{v}=\left(\left(\mathbf{1} / \alpha^{\prime}\right) \times v_{\mathbf{M A X}} \times S\right) /\left(\left(\alpha / \alpha^{\prime}\right) \times K_{\mathbf{M}}+S\right)
$$

where $\alpha$ and $\alpha^{\prime}$ are modifying factors defined as:

$$
\begin{gathered}
\boldsymbol{\alpha}=\mathbf{1}+\left(\boldsymbol{I} / \boldsymbol{K}_{\mathbf{i}}\right) \\
\boldsymbol{\alpha}^{\prime}=\mathbf{1}+\left(\boldsymbol{I} / \boldsymbol{K}_{\mathbf{i}}^{\prime}\right)
\end{gathered}
$$

where $I$ is the concentration of the inhibitor and $K_{\mathrm{i}}$ and $K_{\mathrm{i}}^{\prime}$ are the dissociation constants for the enzyme-inhibitor and enzyme-substrate-inhibitor complexes, respectively. Analogizing into the Monod kinetic, since we are dealing with stimulation of growth and not inhibition, the modifying factors are reciprocated: 


$$
\boldsymbol{Y}=\left(\left(\boldsymbol{\beta}^{\prime}\right) \times \boldsymbol{Y}_{\mathbf{M A X}} \times \boldsymbol{D}\right) /\left((\boldsymbol{\beta} / \boldsymbol{\beta}) \times \boldsymbol{m}_{\mathbf{S}}+\boldsymbol{D}\right)
$$

where $\beta$ and $\beta^{\prime}$ are modifying factors for the stimulatory factor - be that an additional electron donor or an additional carbon source, though what they related to physiologically is unknown, however, one would anticipate they are defined:

$$
\begin{aligned}
\boldsymbol{\beta} & =\mathbf{1}+\left(\boldsymbol{B} / \boldsymbol{K}_{\mathbf{B}}\right) \\
\boldsymbol{\beta}^{\prime} & =\mathbf{1}+\left(\boldsymbol{B} / \boldsymbol{K}_{\mathbf{B}}^{\prime}\right)
\end{aligned}
$$

where $B$ is the concentration of the auxiliary substance and $k_{\mathrm{B}}$ and $k_{\mathrm{B}}^{\prime}$ are probably Monod constant-like parameters for the auxiliary substance. For many auxiliary substances, particularly exogenous sugars and electron donors, this may all simplify down to a single modifying factor $\beta$ defined by a single Monod parameter $k_{\mathrm{B}}$, but there will be more complex scenarios such as that of the above example of "forked" chemolithoautotrophic growth of H. neapolitanus on trithionate, if we consider the latter to be "thiosulfate plus sulfite," where the compound is split into these components and they are oxidized either (the latter) to sulfate via sulfite dehydrogenase (EC 1.8.2.1) or (the former) to sulfate via tetrathionate and/or through protein bound intermediates in the Kelly-Friedrich complex - in both of the latter include sulfite dehydrogenase also; thus the pathways do converge eventually, and all are coupled to the respiratory chain at the level of cytochrome $c$, which is directly oxidized by the terminal oxidase; thus the energy metabolisms of the sulfite and thiosulfate components of trithionate converge at this point.

\subsection{Analogous to Inverted Uncompetitive Inhibition}

As a second example, we can consider the exochemolithoheterotrophic growth of Sagittula stellata $\mathrm{E} 37^{\mathrm{T}}$ on fructose with dimethylsulfide as the auxiliary substance, which is oxidized to dimethylsulfoxide (Boden et al. 2011b); $m_{\mathrm{S}}$ and $\mu_{\mathrm{MAX}}$ do not vary between heterotrophic and exochemolithoheterotrophic growth, but $Y_{\mathrm{MAX}}$ is $14 \%$ higher in the latter situation. Using graphical methods, on a $1 / Y$ versus $1 / \mu$ plot, two parallel lines are seen since $m_{\mathrm{S}}$ is constant, as would be the case in enzymology with an uncompetitive inhibitor $-a$ is increased, $m_{\mathrm{S}}$ stays the same, and $Y_{\mathrm{MAX}}$ is increased. Analogizing the modified Lineweaver-Burk kinetic for uncompetitive (anti-competitive) inhibitors:

$$
1 / v=K_{\mathbf{M}} /\left(\mathbf{v}_{\mathbf{M A X}} \times S\right)+\left(1+\alpha^{\prime}\right) / v_{\mathrm{MAX}}
$$

which we can analogize into the Monod kinetic, reciprocating the modifying factor since we are concerned with stimulation:

$$
1 / Y=m_{\mathrm{S}} \times 1 / \mu+Y_{\mathrm{MAX}} / \beta^{\prime}
$$


This allows us to determine $Y$ on fructose plus dimethylsulfide at any given $\mu$, using $Y_{\mathrm{MAX}}$ and $m_{\mathrm{S}}$ from growth on fructose alone. Using data from Boden et al. (2010), we can determine that $\beta^{\prime}$ is 4805 ; thus $K_{\mathrm{B}}^{\prime}$ is $416 \mathrm{nM}$ - presumably this is a Monod constant for dimethylsulfide and is indeed in the right order of magnitude for the $K_{\mathrm{S}}$ of high-affinity substrates such as oxygen or vitamins (Pirt 1975) - one would anticipate that high affinity was necessary for highly volatile substances such as this. If the organism were grown at a range of concentrations of the auxiliary substance $(B)$, each at a range of $\mu$, the Dixon plot (Dixon 1953) and its associated modifications (Cornish-Bowden 1974) can also be used to determine the magnitude of $K_{\mathrm{B}}^{\prime}$ for mixed metabolic modes that fit the inverted uncompetitive inhibitor kinetic (such as Methylophaga thiooxydans DMS010 ${ }^{\mathrm{T}}$ on methanol/thiosulfate or dimethylsulfide/ thiosulfate, Boden et al. 2010). The precise nature of the interaction of the substrate versus the auxiliary substance at energetic level is not clear; however, it is worth noting that in the examples of $S$. stellata and M. thiooxydans, there is no overlap between the pathways for the main substrate oxidation and for the auxiliary substance oxidation. In the former, fructose is oxidized via glycolysis and Krebs' cycle, with $\mathrm{NAD}(\mathrm{P}) \mathrm{H}$ formation at the former being used to create proton motive force $(\Delta p)$ at the ubiquinone-10-coupled NADH dehydrogenase (EC 1.6.5.3), with addition being generated at the $b c_{1}$ complex from electrons coupled at the level of succinate dehydrogenase and the quinone pool. Dimethylsulfide in some Alphaproteobacteria is oxidized to dimethylsulfoxide via the NADPH coupled trimethylamine monooxygenase (EC 1.14.13.148, Lidbury et al. 2016); however, since NADPH is consumed during this oxidation and there is no further downstream metabolism in S. stellata, this enzyme cannot be responsible for DMS oxidation when acting as an electron donor for energy metabolism. A more likely scenario is a ubiquinone-10 (UQ-10) coupled dimethylsulfoxide reductase (EC 1.8.5.3) acting in the reverse direction, generating ubiquinol-10 $\left(\mathrm{UQH}_{2}-10\right)$, which donates to the $b c_{1}$ complex (EC 1.10.2.2), which in turn donates to cytochrome $\mathrm{c}$, which donates to the $c b b_{3^{-}}$or $a a_{3}$-type cytochrome c oxidases (both proton-translocating, EC 1.9.3.1) found in this genus, followed by reduction of molecular oxygen:

$$
\begin{gathered}
\left(\mathrm{CH}_{3}\right)_{2} \mathrm{~S}+\mathrm{H}_{2} \mathrm{O}+\mathrm{UQ}-\mathrm{HO} \rightarrow\left(\mathrm{CH}_{3}\right)_{2} \mathrm{SO}+\mathrm{UQH}_{2}-10 \\
\mathrm{UQH}_{2}-\mathbf{1 0} \rightarrow b c_{1} \rightarrow c \rightarrow a a_{3} / c b b_{3} \rightarrow \mathrm{O}_{2}
\end{gathered}
$$

Alternatively, as is the case with the same enzyme in Rhodobacter spp., also from the Alphaproteobacteria, the enzyme is coupled to a menaquinone (MK), forming menaquinol $\left(\mathrm{MKH}_{2}\right)$ which donates directly to the proton-translocating $b o_{3}$-type menaquinol oxidase (EC 1.10.3.x), followed by reduction of molecular oxygen:

$$
\begin{aligned}
\left(\mathrm{CH}_{3}\right)_{2} \mathrm{~S}+\mathrm{H}_{2} \mathrm{O}+\mathrm{MK} & \rightarrow\left(\mathrm{CH}_{3}\right)_{2} \mathrm{SO}+\mathrm{MKH}_{2} \\
\mathrm{MKH}_{2} & \rightarrow \mathrm{Bo}_{3} \rightarrow \mathrm{O}_{2}
\end{aligned}
$$

If menaquinone is the site of coupling of electrons from dimethylsulfide to the respiratory chain and the $b o_{3}$ oxidase (versus the $a a_{3}$ and $c b b_{3}$ oxidases used in fructose metabolism) is thus used, there is no overlap between the entire pathway of 
fructose oxidation and that of dimethylsulfide oxidation - thus, the two energy metabolisms - whereas in the previous example of trithionate versus thiosulfate oxidation in H. neapolitanus, the energy metabolisms overlap. A further example of this type of kinetic is observed in Beggiatoa alba ATCC $33555^{\mathrm{T}}$ grown on acetate with or without sulfide as an auxiliary electron donor (Güde et al. 1981).

\subsection{Analogous to Inverted Competitive Inhibition}

The final example we will consider is the analogy of competitive inhibition, which is observed in Acidithiobacillus ferrooxidans grown on tetrathionate as the electron donor and carbon dioxide as the carbon source (Eccleston and Kelly 1978) at 0.03\% $(v / v)$ in air or at $9.00 \%(v / v)$ in air. In this case, the $1 / Y$ versus $1 / \mu$ plot shows two lines that cross at the $y$-intercept, i.e., share the same $Y_{\mathrm{MAX}}(12.5 \mathrm{~g}$ dry biomass $/ \mathrm{mol}$ tetrathionate) but have different gradients $-m_{\mathrm{S}}$ ( $\mathrm{mmol}$ tetrathionate) at the elevated carbon dioxide partial pressure being $0.55 \mathrm{mmol} / \mathrm{g}$.h versus $1.02 \mathrm{mmol} / \mathrm{g} . \mathrm{h}$ at atmospheric levels. These data follow the expected shape of the Lineweaver-Burk plot of a competitive inhibitor but inverted. The modified Michaelis-Menten kinetic for this inhibition is:

$$
1 / v=\left(\left(K_{\mathrm{M}} \times \alpha\right) / v_{\mathrm{MAX}}\right) \times 1 / S+1 / v_{\mathrm{MAX}}
$$

thus for a stimulated growth kinetic:

$$
\mathbf{1} / \boldsymbol{Y}=\left(\left(\boldsymbol{m}_{\mathrm{S}} \times(\mathbf{1} / \boldsymbol{\beta})\right) / \boldsymbol{\mu}+\mathbf{1} / \boldsymbol{Y}_{\mathrm{MAX}}\right.
$$

Therefore, for the Eccleston and Kelly (1978) data, $\beta$ is 1.77 ; thus at $9 \%(v / v)$ carbon dioxide, with the concentration in solution determined by Henry's law, $K_{\mathrm{B}}$ is $43.67 \mu \mathrm{M}$. This presumably represents a Monod constant-like coefficient for the carbon source - carbon dioxide - which is in the same order of magnitude as reported values (Pirt 1975).

\subsection{Analogous to Inverted Noncompetitive Inhibition}

In this scenario, $Y_{\mathrm{MAX}}$ and $m_{\mathrm{S}}$ would be different, but the specific maintenance rate $a$ would be identical (on a $1 / Y$ vs. $1 / D$ plot, the $x$-intercept is $-1 / a$ ) - but this is in part owing to the paucity of chemostat data available for which there are raw data to examine. It is seen in Acidithiobacillus ferrooxidans on thiosulfate versus tetrathionate limitation (Kelly et al. 1987) - in the former condition, $Y_{\text {MAX }}$ was $8.2 \mathrm{~g} / \mathrm{mol}$ and in the latter, $11.9 \mathrm{~g} / \mathrm{mol} ; m_{\mathrm{S}}$ was also different -1.45 and $1.00 \mathrm{mmol} / \mathrm{g} . \mathrm{h}$, respectively. The specific maintenance rate $a$ was 11.9 in both conditions. There is no straightforward set of equations for the derivation of $v$ in enzymology for an inhibited state of this kind working from $v_{\mathrm{MAX}}$ for the uninhibited version. In this example, thiosulfate is oxidized to tetrathionate, and the downstream metabolism is 
largely the same; pace the electrons from that initial oxidation. If we consider tetrathionate as two thiosulfates and redetermine $Y_{\mathrm{MAX}}$, we have $5.95 \mathrm{~g} / \mathrm{mol}$ thiosulfate equivalents. Thus, we could subtract this from $Y_{\text {MAX }}$ for thiosulfate, finding the difference between the two is $2.25 \mathrm{~g} / \mathrm{mol}$ thiosulfate - if the catabolism of exogenous and endogenous tetrathionate were identical and had an identical cost (e.g., transport across membranes, etc.), $2.25 \mathrm{~g}$ dry biomass would be the amount obtained purely from the initial oxidation of $1 \mathrm{~mol}$ of thiosulfate to tetrathionate. Since $m_{\mathrm{S}}$ varies between the two electron donors - albeit not by very much - with tetrathionate having the lower maintenance cost, this could reflect not needing to make and maintain the enzymes of the initial thiosulfate oxidation or the need to undertake two transport steps; thiosulfate is bought into the periplasm and oxidized, and tetrathionate is transported to the cytoplasm for further metabolism; in tetrathionate-grown cells, tetrathionate is taken to the cytoplasm directly, cutting down on transport costs. It is worth noting that thiosulfate was supplied at $20 \mathrm{mM}$ versus $10 \mathrm{mM}$ tetrathionate, meaning the ionic strength when the latter electron donor was used is lower, which could also account for the lower $m_{\mathrm{S}}$ since the osmotic balance costs will be lower.

\section{Conclusions}

In this section we have demonstrated that graphical methods can be useful for determining how different metabolic modes relate - all truly chemolithoheterotrophic growth we observed followed inverted uncompetitive inhibition; changing the carbon source concentration at a fixed electron donor level in autotrophic growth followed inverted competitive inhibition; electron donors that are metabolized largely separately followed inverted mixed inhibition, whereas those metabolized sequentially followed inverted noncompetitive inhibition. With further data and studies that follow the Dixon analysis method (Dixon 1953), it should be possible to determine the factors $\beta$ and $\beta^{\prime}$ and the constants $K_{\mathrm{B}}$ and $K_{\mathrm{B}}^{\prime}$ for a range of organisms and conditions, which will better enable us to understand what these constants mean in physiological terms and how they can be used to better model the effects of mixed metabolism.

\section{Research Needs}

- Determination of chemostat kinetic parameters $Y_{\mathrm{MAX}}, m_{\mathrm{S}}, Y_{\mathrm{ATP}}, m_{\mathrm{ATP}}, a, E_{\mathrm{G}}, Y_{\mathrm{O}}$, $K_{\mathrm{S}}$, etc., for a wider range of organisms and growth conditions, using the latest technologies for more precise data

- Where raw data are available or can be extracted from plots, a reanalysis of older studies using hyperbolic fitting to yield more precise data

- A move to the determination of more meaningful growth parameters in batch and continuous culture - specifically dry weight or total organic carbon - and an end to the publication of raw optical densities without proper analysis 
Acknowledgments RB thanks those who originally taught him the mathematical basis of chemostat kinetics that inspired him in the first place - Professor Jeremy Mason and Dr. Ann P Wood (both formerly of King's College London, London, UK) - and those from whom he learnt the practicalities, Professor Donovan P Kelly (Professor Emeritus, University of Warwick, Coventry, UK), Dr. Elena Borodina (Lecturer in Science, Weston College of Further and Higher Education, Weston-super-Mare, UK), Mr. Gez Chapman, Mrs. Jane Green, and the late Dr. Steve Stanley (all formerly of University of Warwick, Coventry, UK). LPH and RB both thank our collaborators Dr. Kathleen M Scott (Associate Professor, University of South Florida, Tampa, FL, USA) and Dr. Jan Kuever (Head of Department of Microbiology, Official Material Testing Institute of the Free Hanseatic City of Bremen, Germany) for continued stimulating discussions on continuous culture methodology and kinetics and the wider field of growth physiology.

\section{References}

Anthony C (1982) The biochemistry of methylotrophs. Academic, London

Aronoff S (1957) Techniques of radiobiochemistry. The Iowa State College Press, Ames

Avery GB Jr, Shannon RD, White JD, Martens CS, Alperin MJ (1999) Effect of seasonal changes in the pathways of methanogenesis on the $\partial^{13} \mathrm{C}$ values of pore water methane in a Michigan peatland. Global Biogeochem Cycles 13:475-484

Bauchop T, Elsden SR (1960) The growth of micro-organisms in relation to their energy supply. J Gen Microbiol 23:457-469

Boden R, Murrell JC (2011) Response to mercury (II) ions in Methylococcus capsulatus (bath). FEMS Microbiol Lett 324:106-110

Boden R, Kelly DP, Murrell JC, Schäfer H (2010) Oxidation of dimethylsulfide to tetrathionate by Methylophaga thiooxidans sp. nov.: a new link in the sulfur cycle. Environ Microbiol 12:2688-2699

Boden R, Borodina E, Wood AP, Kelly DP, Murrell JC, Schäfer H (2011a) Purification and characterization of dimethylsulfide monooxygenase from Hyphomicrobium sulfonivorans. J Bacteriol 193:1250-1258

Boden R, Murrell JC, Schäfer H (2011b) Dimethylsulfide is an energy source for the heterotrophic marine bacterium Sagittula stellata. FEMS Microbiol Lett 322:188-193

Boden R, Hutt LP, Huntemann M, Clum A, Pillay M, Palaniappan K, Varghese N, Mikhailova N, Stamatis D, Reddy T, Ngan CY, Daum C, Shapiro N, Markowitz V, Ivanova N, Woyke T, Kyrpides N (2016) Permanent draft genome of Thermithiobacillus tepidarius DSM 3134T, a moderately thermophilic. Stand Genomic Sci 11:74

Borodina E, Kelly DP, Rainey FA, Ward-Rainey NL, Wood AP (2000) Dimethylsulfone as a growth substrate for novel methyl-tropic species of Hyphomicrobium and Arthrobacter. Arch Microbiol $173: 425-437$

Chongcharoen R, Smith TJ, Flint KP, Dalton H (2005) Adaptation and acclimatization to formaldehyde in methylotrophs capable of high-concentration formaldehyde detoxification. Microbiology 151:2615-2622

Cohen A (1922) Xylenol blue and its proposed use as a new and improved indicator in chemical and biochemical work. Biochem J 16:31-34

Cooper CM, Fernstorm GA, Miller SA (1944) Performance of agitated gas-liquid contactors. Ind Eng Chem 36:504-509

Cornish-Bowden A (1974) A simple graphical method for determining the inhibition constants of mixed, uncompetitive and non-competitive inhibitors. Biochem J 137:143-144

Dixon M (1953) The determination of enzyme inhibitor constants. Biochem J 55:170-171

Drevon D, Fursa SR, Malcolm AL (2016) Intercoder reliability and validity of WebPlotDigitizer in extracting graphed data. Behav Modif 41:323-339

Eccleston M, Kelly DP (1978) Oxidation kinetics and chemostat growth kinetics of Thiobacillus ferrooxidans on tetrathionate and thiosulfate. J Bacteriol 134:718-727 
Gao P, Sun C, Li Y, Zou X, Wu X, Ling Y, Luan C, Chen H (2017) Vital staining of bacteria by sunset yellow pigment. Pol J Microbiol 66:113-117

Garcia-Ochoa F, Gomez E (2009) Bioreactor scale-up and oxygen transfer rate in microbial processes: an overview. Biotechnol Adv 27:153-176

Good NE, Izawa S (1972) Hydrogen ion buffers. Methods Enzymol 24:53-68

Good NE, Winget DG, Winter W, Connolly TN, Izawa S, Singh RMM (1966) Hydrogen ion buffers for biological research. Biochemistry 5:467-477

Grady JK, Chasteen ND, Harris DC (1988) Radicals from "Good's" buffers. Anal Biochem 173:111-115

Groisman A, Lobo C, Cho H, Campbell JK, Dufour YS, Stevens AM, Levchenko A (2005) A microfluidic chemostat for experiments with bacterial and yeast cells. Nat Methods 2:685-589

Güde H, Strohl WR, Larkin JM (1981) Mixotrophic and heterotrophic growth of Beggiatoa alba in continuous culture. Arch Microbiol 129:357-360

Haldane JBS (1957) Graphical methods in enzyme chemistry. Nature 179:832-832

Hanes CS (1932) Studies on plant amylases: the effect of starch concentration upon the velocity of hydrolysis by the amylase of germinated barley. Biochem J 26:1406-1421

Harrison DEF, Loveless JE (1971) The effect of growth conditions on respiratory activity and growth efficiency in facultative anaerobes in chemostat culture. J Gen Microbiol 68:35-43

Harrison DEF, MacLennan DG, Pirt SJ (1969) Responses of bacteria to dissolved oxygen tension. In: Perlman D (ed) Fermentation advances. Academic, New York, pp 117-143

Hofstee BHJ (1959) Non-inverted versus inverted plots in enzyme kinetics. Nature 184:1296-1298

Hutt LP (2016) Taxonomy, physiology and biochemistry of the sulfur bacteria. PhD thesis, University of Plymouth

Karagouni AD, Slater JH (1978) Growth of the blue-green alga Anacystis nidulans during washout from light- and carbon dioxide-limited chemostats. FEMS Microbiol Lett 4:295-299

Kelly DP, Syrett PJ (1964) The Effect of Uncoupling Agents on Carbon Dioxide Fixation by a Thiobacillus. J Gen Microbiol 34(2):307-317

Kelly DP (1965) Energy metabolism of the chemoautotrophic bacterium, Thiobacillus. PhD thesis, University College London

Kelly DP, Syrett PJ (1963) Effect of 2:4-dinitrophenol on carbon dioxide fixation by a Thiobacillus. Nature 197:1087-1089

Kelly DP, Syrett PJ (1966) Energy coupling during sulphur compound oxidation by Thiobacillus sp. strain c. J Gen Microbiol 43:109-118

Kelly DP, Wood AP (1994) Synthesis and determination of polythionates and thiosulfate. Methods Enzymol 243:475-501

Kelly DP, Wood AP (1998) Microbes of the sulfur cycle. In: Burlage R, Atlas R, Stahl D, Geesey G, Gayler G (eds) Techniques in microbial ecology. Oxford University Press, New York

Kelly DP, Mason J, Wood AP (1987) Energy metabolism in chemolithotrophs. In: van Verseveld HW, Duine JA (eds) Microbial growth on $\mathrm{C}_{1}$ compounds. Proceedings of the 5th international symposium. Martinus Nijhoff Publishers, Dordrecht

Koch AL (1981) Growth measurement. In: Gerhardt P, Murray RGE, Costilow RN, Nester EN, Wood WA, Krieg NR, Phillips GB (eds) Manual of methods for general bacteriology. American Society for Microbiology, Washington, pp 182-207

Lidbury I, Kröber E, Zhang Z, Zhu Y, Murrell JC, Chen Y, Schäfer H (2016) A mechanism for bacterial transformation of dimethylsulfide to dimethylsulfoxide: a missing link in the marine organic sulfur cycle. Environ Microbiol 18:2753-2766

Lineweaver H, Burk D (1934) The determination of enzyme dissociation constants. J Am Chem Soc 56:658-666

Long Z, Nugent E, Jager A, Cicuta P, Sclavi B, Consentino Lagomarsino M, Dorfman KD (2013) Microfluidic chemostat for measuring single cell dynamics. Lab Chip 13:947-945

Lyons D, Nickless G (1968) The lower oxy-acids of sulphur. In: Nickless G (ed) Inorganic sulphur chemistry. Elsevier, Amsterdam, pp 509-534 
MacLennan DG, Ousby JC, Vasey RB, Cotton NT (1971) The influence of dissolved oxygen on Pseudomonas AM1 grown on methanol in continuous culture. J Gen Microbiol 69:395-404

Mason J (1986) Microbial growth and the oxidation of inorganic sulphur compounds. PhD thesis, University of Warwick

Michaelis L, Menten ML (1913) Die Kinetik der Invertinwirkung. Biochem Z 49:333-339

Monod J (1942) Recherches sur la croissance des Cultures Bactériennes. Hermann, Paris

Monod J (1949) The growth of bacterial cultures. Annu Rev Microbiol 3:371-394

Pirt SJ (1965) The maintenance energy of bacteria in growing cultures. Proc R Soc B 163:224-231

Padden AN (1997) Microbial degradation of organic sulfur compounds. PhD thesis, King's College London

Padden AN, Kelly DP, Wood AP (1998) Chemolithoheterotrophy and mixotrophy in the thiophene2-carboxylic acid-utilizing Xanthobacter tagetidis. Arch Microbiol 169:249-256

Parkhurst DL, Appelo CAJ (1999) User's guide to PHREEQC (Version 2) - a computer program for speciation, batch-reaction, one-dimensional transport, and inverse geochemical calculations: U. S. Geological Survey Water-Resources investigations report 99-4259

Pirt SJ (1975) Principles of microbe and cell cultivation. Halsted Press, New York

Pirt SJ, Callow DS (1958) Observations on foaming and its inhibition in a bacterial culture. J Appl Microbiol 21:211-216

Pirt SJ, Callow DS (1960) Studies on the growth of Penicillium chrysogenum in continuous flow culture with reference to penicillin production. J Appl Bacteriol 23:87-98

Pirt SJ, Panikov N, Lee Y-K (1979) The miniloop: a small-scale air-lift microbial culture vessel and photo biological reactor. J Chem Technol Biotechnol 29:437-441

Pogliano J, Osborne N, Sharp MD, Abanes-De Mello A, Perez A, Sun Y-L, Pogliano K (1999) A vital stain for studying membrane dynamics in bacteria: a novel mechanism controlling septation during Bacillus subtilis sporulation. Mol Microbiol 31:1149-1159

Raffo M (1908) Ueber kolloiden Schwefel. Kolloid Z 2:358-360

Reasoner DJ, Geldreich EE (1985) A new medium for the enumeration and subculture of bacteria from potable water. Appl Environ Microbiol 49:1-7

Skidmore DW (1979) Purification of carbon disulphide for use as a solvent in gas chromatography. Ann Occup Hyg 22:181-182

Smith PK, Krohn RI, Hermanson GT, Mallia AK, Gartner FH, Provenzano MD, Fujimoto EK, Goeke NM, Olson BJ, Klenk DC (1985) Measurement of protein using bicinchoninic acid. Anal Biochem 150:76-85

Solórzano L (1969) Determination of ammonia in natural waters by the phenol hypochlorite method. Limnol Oceanogr 14:799-801

Soni ML, Kapoor RC (1981) Some thermodynamic parameters for hydroxyl amino acids: bicine and tricine. Int J Quantum Chem 20:385-391

Steudel R (2003) Aqueous sulfur sols. In: Steudel R (ed) Elemental sulfur and sulfur-rich compounds I. Springer, Berlin, pp 156-166

Stouthamer AH (1973) A theoretical study on the amount of ATP required for synthesis of microbial cell material. Anton van Leeuwenhoek 39:545-565

Stouthamer AH (1976) Yield studies in microorganisms, Patterns of Progress PP/M/3. Meadowfield, Shildon

Stouthamer AH, Bettenhausen C (1973) Utilization of energy for growth and maintenance in continuous and batch cultures of microorganisms. Biochim Biophys Acta 301:53-70

Tuovinen OH, Kelly DP (1973) Studies on the growth of Thiobacillus ferrooxidans I. Use of membrane filters and ferrous iron agar to determine viable numbers, and comparison with ${ }^{14} \mathrm{CO}_{2}$-fixation and iron oxidation as measures of growth. Arch Mikrobiol 88:285-298

van Steveninck J, Booij HL (1964) The role of polyphosphates in the transport mechanism of glucose in yeast cells. J Gen Physiol 48:43-60

von Bodegom P (2007) Microbial maintenance: a critical review on its quantitation. Microb Ecol 53:513-523 
von Weimarn PP (1926) Über schwefellsungen von allen furben de spektrums. Kolloidchemische Beiheffe band XXII. Booklet 1-2

Watson TG (1970) Effects of sodium chloride on steady-state growth and metabolism of Saccharomyces cerevisiae. J Gen Microbiol 64:91-99

Wood AP, Kelly DP (1981) Mixotrophic growth of Thiobacillus A2 in chemostat culture on formate and glucose. J Gen Microbiol 125:55-62

Wood AP, Kelly DP (1986) Chemolithotrophic metabolism of the newly-isolated moderately thermophilic, obligately autotrophic Thiobacillus tepidarius. Arch Microbiol 144:71-77

Xia R (2016) The dying breed of craftsmen behind the tools that make scientific research possible. LA Times, 25 June 2016. http://www.latimes.com/local/education/la-me-caltech-glassblower20160613-snap-story.html 